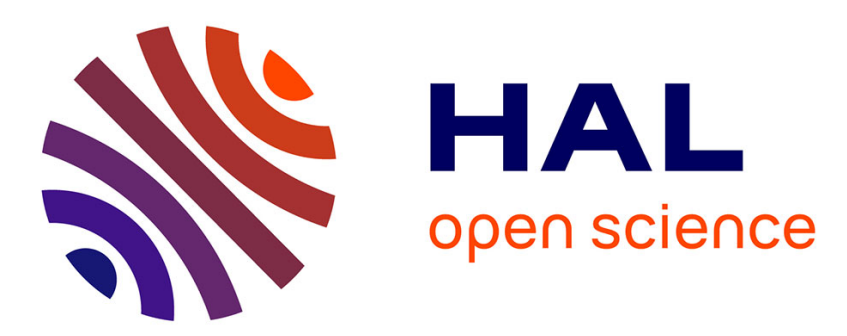

\title{
Morphology and structure of A-amylose single crystals
}

Jean-Luc Putaux, Nicole Montesanti, Gabrielle Veronese, Alain Buleon

\section{To cite this version:}

Jean-Luc Putaux, Nicole Montesanti, Gabrielle Veronese, Alain Buleon. Morphology and structure of A-amylose single crystals. Polymer, 2011, 52 (10), pp.2198 - 2205. 10.1016/j.polymer.2011.03.014 . hal-02646108

\section{HAL Id: hal-02646108 \\ https: / hal.inrae.fr/hal-02646108}

Submitted on 30 Nov 2021

HAL is a multi-disciplinary open access archive for the deposit and dissemination of scientific research documents, whether they are published or not. The documents may come from teaching and research institutions in France or abroad, or from public or private research centers.
L'archive ouverte pluridisciplinaire HAL, est destinée au dépôt et à la diffusion de documents scientifiques de niveau recherche, publiés ou non, émanant des établissements d'enseignement et de recherche français ou étrangers, des laboratoires publics ou privés. 


\section{Morphology and structure of A-amylose single crystals}

Jean-Luc Putaux ${ }^{a *}$, Nicole Montesanti ${ }^{a}$, Gabrielle Véronèse $^{\mathrm{b}, c}$, Alain Buléon $^{\mathrm{d}}$

a Centre de Recherches sur les Macromolécules Végétales (CERMAV-CNRS), BP 53, F-38041 Grenoble Cedex 9, France - affiliated with Université Joseph Fourier and member of the Institut de Chimie Moléculaire de Grenoble.

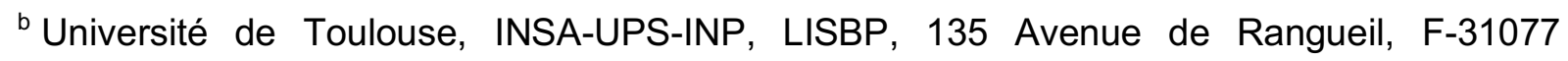
Toulouse, France

c UMR 5504, UMR 792 Ingénierie des Systèmes Biologiques et des Procédés, CNRS, INRA, F-31400 Toulouse, France

d UR1268 Biopolymères Interactions Assemblages, INRA, F-44300 Nantes, France

*corresponding author: jean-luc.putaux@cermav.cnrs.fr

Published in: Polymer 52 (2011), 2198-2205

DOI: 10.1016/J.POLYMER.2011.03.014 

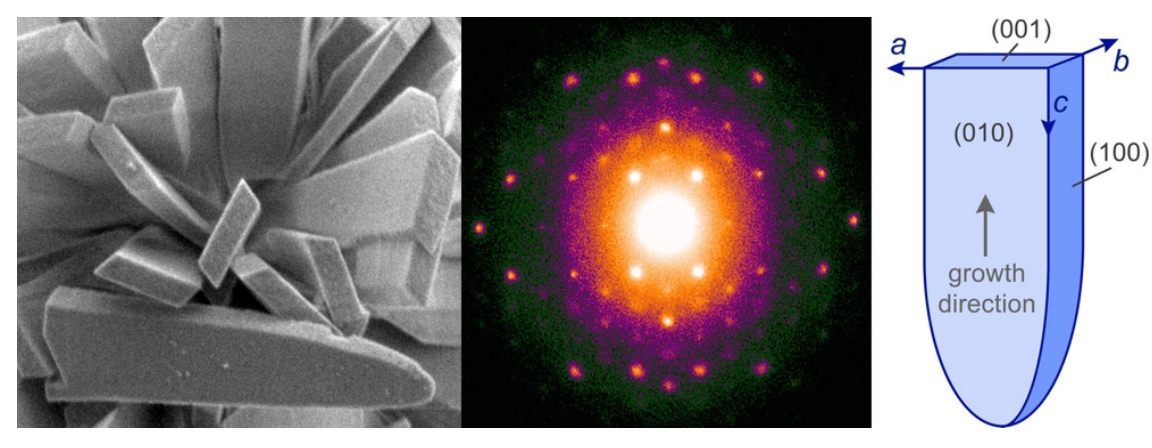

\begin{abstract}
A-type single crystals were prepared in dilute water/acetone solutions by crystallizing narrow fractions of short amylose chains biosynthesized in vitro. The $5 \mu \mathrm{m}$-long spindle-shaped crystals were observed by scanning and transmission electron microscopy. Electron diffraction patterns were recorded from frozen-hydrated crystals at different tilt angles around selected crystallographic axes. In particular, a series of patterns corresponding to [hk0] zone axes was collected by rotation around the long dimension of the crystal. A description of the crystal habit was proposed by correlating the electron diffraction data with the microscopy images. The double helices are oriented along the long dimension of the crystal (c-axis) and packed in a parallel fashion into lamellae with a parallelogram cross-section defined by the $a$ and $b$ axes of the monoclinic unit cell. A-amylose single crystals are polar objects that grow in the direction of the reducing end of the amylose chains, and the $c$-axis is oriented opposite to the growth direction.
\end{abstract}

Keywords: A-amylose, single crystals, electron diffraction, polar crystal, synthetic amylose 


\section{Introduction}

Amylose, the linear constituent of starch, consisting of $\alpha(1,4)$-linked D-glucopyranosyl units, can be recrystallized in vitro into the so-called $A$ and $B$ allomorphs that also occur in the native granules. Typically, A-type starch is found in cereals while B-starch frequently occurs in tubers and amylose-rich starches [1]. The determination of the distribution and size of crystalline domains in starch granules is difficult due to their complex multilayered ultrastructure and low crystallinity. Crystals grown in vitro have thus been used to collect high resolution diffraction data and propose molecular models that were transposed to the crystalline domains of native starch granules.

A three-dimensional model of the A allomorph was first proposed by Wu and Sarko using fiber X-ray diffraction data collected from stretched films of long-chain amylose. In this model, amylose was crystallized as parallel-stranded right-handed 6-fold double helices organized in an antiparallel fashion in an orthorhombic unit cell [2]. A-type single crystals were prepared by Buléon et al. by addition of ethanol to a hot dilute aqueous solution of short amylose chains stemming from acid hydrolysis of potato starch with an average degree of polymerization $\overline{D P}$ of 15 [3]. For the first time, electron diffraction patterns were recorded from frozen-hydrated crystals but the collected data did not allow to confirm the symmetry and unit cell proposed by Wu and Sarko. Spindle-shaped crystals were also prepared by Imberty et al. from potato starch dextrins by diffusion of acetone vapors at $60{ }^{\circ} \mathrm{C}[4,5]$. The general crystal morphology was described from transmission electron microscopy (TEM) images and from electron diffraction patterns of frozen-hydrated specimens, the authors deduced the axial orientation of the $c$-axis of the unit cell. After analysis of a set of electron diffraction patterns recorded at different crystal tilts, and reindexing of Wu and Sarko's X-ray diffraction patterns, an improved structure was proposed, combining conformational analysis and X-ray refinement. The revised model was based on a monoclinic unit cell containing parallel 6-fold left-handed double helices and 4 water molecules per unit cell [5]. More recently, Popov et al. confirmed the monoclinic nature of the unit cell using synchrotron X-ray microdiffraction data recorded from single crystals prepared from amylose biosynthesized in vitro [6]. The structure was refined using high resolution diffraction data sets collected from several single crystals and the resulting model allowed to describe new fine details of A-amylose [7]. In particular, the double helices were distorted in order to accommodate intra-crystalline pockets of water molecules located between the helices. The total amount of water in the crystal was found to be twice that proposed by Imberty et al. [5].

In a previous work, we have studied the influence of chain length, polydispersity and branching degree of short-chain amylose on the morphology of A-type crystals prepared from dilute aqueous solutions, at $60^{\circ} \mathrm{C}$, in the presence of acetone [8]. In particular, rosettes or fan- 
like assemblies of 5-10 $\mu \mathrm{m}$-long spindle-shaped crystals were obtained from narrow fractions of amylose synthesized in vitro from sucrose by the recombinant amylosucrase from Neisseria polysaccharea. The morphological analysis of the single crystals combined with preliminary electron diffraction data showed that the growth direction coincided with but was opposite to the $c$-axis of the monoclinic unit cell. The axial growth of A-amylose thus clearly appeared to be favored unlike in the case of most polymer single crystals [8].

In the present complementary paper, we provide the first detailed description of the habit of faceted A-amylose single crystals based on an extensive electron diffraction analysis carried out in relation with the crystal morphology determined from scanning and transmission electron microscopy images. We have indexed the growing faces and determined the polarity of the amylose chains with respect to the growth direction.

\section{Experimental section}

\subsection{Preparation of the synthetic amylose fractions}

Linear $\alpha(1,4)$-D-glucan chains were synthesized in vitro by the purified recombinant amylosucrase from Neisseria polysaccharea, as described elsewhere [8]. The synthesis reaction was performed at $30^{\circ} \mathrm{C}$ during $24 \mathrm{~h}$ in a $50 \mathrm{mM}$ Tris- $\mathrm{HCl}$ buffer at $\mathrm{pH} 7.0$, from $600 \mathrm{mM}$ sucrose as glucosyl donor and $100 \mathrm{mM}$ maltose as acceptor, using $0.5 \mathrm{U} / \mathrm{mL}$ glutathione-Stransferase/amylosucrase. The resulting products (fructose and maltooligosaccharides of $D P$ ranging from 2 to 36 ) were subsequently fractionated by preparative gel filtration using a $480 \mathrm{~mL}$ column of Biogel P6 Extra Fine (Biorad). Sugars were eluted at $60{ }^{\circ} \mathrm{C}$ in water using a flow-rate of $0.66 \mathrm{~mL} / \mathrm{min}$. High-performance anion-exchange chromatography with pulsed amperometric detection (HPAEC-PAD) was used to determine the DP distribution of each resulting fraction of short amylose chains. In the following, these fractions are referred to as SAA (for "synthetic amylose amylosucrase"), associated to their minimum and maximum $D P$, respectively. As described in details in Supplementary Material, the fractions are characterized by their number- and weight-average mean $D P \mathrm{~s}\left(\overline{D P_{n}}\right.$ and $\overline{D P_{w}}$, respectively $)$ and polydispersity index $P=\overline{D P_{w}} / \overline{D P_{n}}$ [8]. Since for the electron crystallography study, it was necessary to select crystals with a clearly faceted shape and a thickness not exceeding $200 \mu \mathrm{m}$, only two fractions have been considered: SAA12-23 $\left(\overline{D P_{n}}=16.4, \overline{D P_{w}}=16.7, P=1.013\right)$ and SAA17-28 $\left(\overline{D P_{n}}\right.$ $\left.\overline{D P_{n}}=20.9, \overline{D P_{w}}=21.0, P=1.006\right)$.

\subsection{Crystallization}

The protocol was adapted from the procedures described by Buléon et al. [3] and Imberty et al. [5]. Aqueous dispersions of amylose $(0.05 \% \mathrm{w} / \mathrm{v})$ were submitted to nitrogen bubbling for $20 \mathrm{~min}$ and heated in sealed vials at $150{ }^{\circ} \mathrm{C}$ for $15 \mathrm{~min}$. The solutions were then cooled down 
to $80{ }^{\circ} \mathrm{C}$, filtered through $0.2 \mu \mathrm{m}$ pre-heated Sartorius Minisart RC 25 filters, heated again at $150{ }^{\circ} \mathrm{C}$ for $15 \mathrm{~min}$, cooled down to $80^{\circ} \mathrm{C}$ and poured in a three-neck $100 \mathrm{~mL}$ glass balloon preheated at $55{ }^{\circ} \mathrm{C}$ in an oil bath. Acetone was heated at $60{ }^{\circ} \mathrm{C}$ in a separate compartment and the resulting vapors diffused through pre-heated glass tubes into the amylose solution under gentle stirring. Upon crystallization, the mixtures were allowed to slowly cool down to room temperature. All specimens were kept in their water-acetone mother liquor and stored at $4{ }^{\circ} \mathrm{C}$.

\subsection{Scanning electron microscopy (SEM)}

Drops of dilute crystal suspensions were allowed to dry onto copper stubs. The specimens were coated with Au/Pd and observed in secondary electron mode with a JEOL JSM-6100 scanning electron microscope operating at $8 \mathrm{kV}$. A second group of specimens were coated with a $2 \mathrm{~nm}$-thick layer of $\mathrm{Pt} / \mathrm{Pd}$ and secondary electron images were recorded with a JEOL JSM-7000F microscope equipped with a field emission gun and operating at $1.5 \mathrm{kV}$.

\subsection{Transmission electron microscopy (TEM) and electron diffraction}

Drops of dilute crystal suspensions were deposited onto glow-discharged carbon-coated copper grids and the liquid in excess was blotted with filter paper. The specimens were rapidly mounted on a Gatan 626 specimen holder, quench-frozen in liquid nitrogen, transferred into the microscope and cooled down to $-180^{\circ} \mathrm{C}$. The crystals were observed at low temperature, in low illumination conditions, using a Philips CM200 'Cryo' microscope operating at $80 \mathrm{kV}$ for imaging and $200 \mathrm{kV}$ for diffraction. Electron diffraction diagrams were recorded on $1 \mu^{2}$ circular areas of single crystals that were tilted around selected reciprocal axes. For calibration purpose, crystals were deposited onto gold-coated TEM carbon films and the diffraction spots were calibrated at low temperature using the diffraction rings of gold. The images were recorded on Kodak SO163 films and the diffraction patterns on Fujifilm imaging plates, read with a Fujifilm BAS-1800II bioimaging analyzer. After indexation and calibration of the diffraction spots, the unit cell parameters were calculated using a least-squares program.

\section{Results}

SEM images of the crystals prepared from fractions SAA12-23 and SAA17-28 are shown in Figures $1 \mathbf{a , b}$ and 1c,d, respectively. In both cases, the crystals are $5 \mu \mathrm{m}$-long, $200 \mathrm{~nm}$-thick and about $1 \mu \mathrm{m}$-wide spindle-shaped platelets with a flat apical end. In addition, FEG-SEM images show the layered structure of the crystals and the constituting lamellae lie perpendicular to the growth axis (Figure 2a). For both samples, the characteristic shape of the crystal cross-section is a parallelogram (Figures $\mathbf{1 b}$ and $\mathbf{1 d}$ ) whose orientation is always the same with respect to the crystal long axis (Figure $\mathbf{2 b}$ ). The obtuse angle between the faces is 
about $120^{\circ}$. As this value is close to that of the angle between the $a$ and $b$ axes of the monoclinic unit cell (about $122^{\circ}$ ), two hypotheses can be made: i) the parallelogram section is a projection of the $(a, b)$ base plane (Figure $2 \mathrm{c}$ ); ii) the widest face contains the a-axis (the aparameter of the unit cell being larger than the $b$-parameter) [5]. These assumptions led us to orient the three $a, b$ and $c$ axes as described in Figure $\mathbf{2 b}$. The $c$-axis would thus point in a direction opposite to the growth direction of the crystal.

Diffraction diagrams were recorded from single crystals lying flat on the carbon film without tilting the specimen holder (Figure 3a). Two equiprobable patterns were observed that were compared to those reported by Imberty et al. [5] as corresponding to the [130] and [140] zone axes of the monoclinic unit cell (Figures $\mathbf{3 b}$ and $\mathbf{3 c}$, respectively). We have thus used these patterns as references to rapidly select crystals for further tilt experiments.

Using the previous hypotheses on the orientation of the $a, b$ and $c$ axes with respect to the crystals, we have calculated a priori the tilt angles around the $c$-axis that would allow reaching a number of low-index [ $h k 0$ ] zone axes of the monoclinic unit cell. The corresponding diagram is presented in Figure 4. The cross-section of the crystal, defined by the $a$ and $b$ axes in real space, has been superimposed on the associated reciprocal lattice. One can see that when a crystal is lying flat on the carbon film at a $0^{\circ}$ tilt angle, the axis perpendicular to its surface is not a low-index zone axis and is located between the [130] and [140] axes. This explains why both diffraction patterns shown in Figure 3 were equiprobable: depending on slight local tilts of the supporting carbon film (within a $\pm 5^{\circ}$ range), the crystal may have been oriented in such a way that the [130] or [140] axis was parallel to the electron beam.

Crystals with their long axis oriented parallel to the tilt axis of the specimen holder were selected for tilt experiments. Two approaches were used to record diffraction patterns. On the one hand, thanks to the high sensitivity of the imaging plates, the electron dose could be reduced in such a way that up to five diffraction patterns could be consecutively recorded from the same crystal at different tilt angles. In general, the last two patterns did not extend to a high resolution due to the accumulated radiation damage but the remaining spots were sufficient to identify the corresponding zone axis. On the other hand, we directly tilted the crystals at specific angles (within a $\pm 35^{\circ}$ range) and recorded unique high-resolution patterns with a higher electron dose. These experiments allowed us to collect the main patterns corresponding to low-index zone axes and establish their order of appearance as a function of the positive or negative angle around the tilt axis.

Two series of diffraction patterns sequentially recorded at selected tilts around the crystal long axis are shown in Figure S1. The characteristic shape of the crystals, with distinct sharp and flat ends, was used to select crystals oriented parallel to the tilt axis of the specimen holder but pointing in two opposite directions. The initial patterns, recorded with an untilted specimen holder (i.e., $0^{\circ}$ ), correspond to the [140] zone axis. The [130] and [120] zone axes were reached 
with relative tilt angles of $\pm 10^{\circ}$ and $\pm 30^{\circ}$, respectively, but the series were recorded by tilting the specimen anticlockwise in one case and clockwise in the other (Figure S1a and S1b, respectively). By using the scheme in Figure 4, the $c$-axis could thus be unambiguously oriented as pointing opposite to the growth direction.

In all patterns, the two main axes were perpendicular, meaning that the $\alpha$ and $\beta$ angles of the unit cell were $90^{\circ}$ and that a triclinic structure could thus be discarded. In addition, Imberty et al. had shown that the patterns recorded at opposite tilt angles around the $c$-axis with respect to the [010] zone axis were different, allowing to discard an orthorhombic structure [5]. We could not reproduce this experiment since the large tilt angles that were required could not be reached with our set-up. From these two results, it was deduced that the unit cell was monoclinic.

Figure 5 a shows a series of TEM images of one crystal rotated around the $c$-axis. A slight axial curvature was observed when the tilt angle exceeded $\pm 20^{\circ}$. Since the crystals were frozen-hydrated before their introduction in the microscope, we do not believe that this curvature was induced by dehydration due to drying and it rather might be a morphological feature that will require further investigation. The contrast variation at the edges of the crystal could also be interpreted considering the parallelogram shape of its cross-section (Figure 5b). By drawing the projection of the reciprocal lattice associated to the crystal at each tilt angle, we have identified the zone axis parallel to the electron beam for each image (Figure 5c). In particular, the contrast of the crystals was uniform and its edges appeared to be sharp when its orientation was such that the [010] axis was parallel to the electron beam.

The combination of the results obtained from Figure $\mathbf{5}$ and Figure $\mathbf{S 1}$ allowed us to unambiguously assign a zone axis to each [hk0] diffraction pattern and fully index the reflections. Four typical patterns corresponding to the [010], [140], [130] and [110] zone axes are displayed in Figure 6 along with the associated spot indexation and orientation of both reciprocal lattice and crystal cross-section. Additional patterns corresponding to [160], [250] and [120] zone axes are shown in Figure S2.

Several diffraction patterns corresponding to zone axes which did not belong to the [hk0] series were also recorded, mostly from crystals prepared from fraction SAA12-23. It is likely that the crystals were supported by a deformed carbon film that was tilted even when the specimen holder was not. As it proved difficult to identify the indices of the zone axes by exploring the reciprocal lattice, we have simulated a series of diffraction diagrams corresponding to low-index zone axes using the atom coordinates given by Popov et al. [7] and the Cerius ${ }^{2}$ software [9]. The resulting patterns were compared to the experimental ones. This revealed that most zone axes belonged to the [ $1 k 1]$ series recorded by tilting the crystals around the [101] axis. The diagrams corresponding to [121], [131], [141] and [161] zone axes are displayed in Figure S3. Two additional patterns, shown in Figure S4, recorded by randomly titling the crystals, could be indexed as corresponding to [021] and [143] zone axes. 
All indexed diffraction patterns were calibrated using a gold standard. Merging the data resulted in a list of 183 independent reflections (Table S1), extending to a $0.1 \mathrm{~nm}$ resolution. The unit cell parameters were calculated from the whole data set using a least-squares regression and imposing a monoclinic constraint: $a=2.098 \mathrm{~nm}, b=1.147 \mathrm{~nm}$ and $c=1.062$ $\mathrm{nm}, \gamma=121.46^{\circ}$. These values agree with those reported by Imberty et al. [5] and Popov et al. [7], determined from electron or X-ray diffraction data (Table 1). However, they match more closely the cell parameters of Popov et al., determined from X-ray microdiffraction data collected at low temperature $\left(-173^{\circ} \mathrm{C}\right)$.

\section{Discussion}

The thorough structural and morphological analyses carried out from electron diffraction data and electron microscopy images allowed us to propose an accurate description of the crystal habit of A-amylose single crystals. The main features are summarized in Figure 7.

\subsection{Morphology}

A-amylose single crystals prepared from short amylose chains, typically with $12<\mathrm{DP}<30$, are thick lamellar platelets [8], the lamellae lying perpendicular to the growth direction. The electron diffraction data confirmed the monoclinic unit cell proposed by Imberty et al. [5] and Popov et al. [7], with the $c$-axis oriented in a direction parallel but opposite to that of the crystal growth. We have unambiguously identified the facets of the crystal: the widest one is the (010) face which contains the a-axis. The thinnest face is $(100)$ and contains the $b$-axis. The flat apical end is the (001) face. The parallelogram cross-section of the crystal thus corresponds to a projection of the $(a, b)$ plane. According to the 3D model proposed by Popov et al. [7], the amylose double helices have a "parallel-down" orientation in the unit cell. The non-reducing end of the chains would thus point toward the $c$-axis and opposite to the growth direction (Figure 7).

The orientation of the A-amylose unit cell in the crystal differs from that observed in lamellar polymer crystals prepared from dilute solutions, for which the $c$-axis is perpendicular to the lamella base plane. In particular, this is the case for several polysaccharides such as cellulose [10-12], chitin [12], or the $V$ form of amylose crystallized in the presence of various complexing agents [1]. Lamellar crystals have also been prepared from numerous synthetic polymers [13]. The axial orientation of the chains in the spindle-shaped A-amylose crystals thus appears to be a rather unique feature. In some aspects, this case has similarities with that of the semicrystalline collagen fibrils prepared in vitro from dilute [14] or concentrated [15] solutions. These fibrils are formed by axial growth of an assembly of triple helices, organized in a quasihexagonal lattice, and exhibit parabolic ends $[16,17]$. Although the crystalline nature and the molecular packing structure of collagen fibrils is still debated, it may be interesting to explore this analogy in further details. 
The reason of the favored axial growth of A-amylose crystals is still unknown. Several observations and hypotheses were discussed in our previous paper in view of classical polymer growth mechanisms [8]. Further investigations will consist in evaluating the influence of operating parameters such as amylose concentration, nature of the precipitant, crystallization temperature and time on the crystal morphology and growth.

\subsection{Polarity}

Since its constituting helices point in the same direction in the unit cell, an A-amylose single crystal can thus be described as a polar object, having reducing and non-reducing edges that would correspond to the apical and nucleation ends, respectively (Figure 7). Chemical polarity in a solid can be desired to confer specific properties, in particular optical and electronic ones, to materials. Several papers describe the in vitro elaboration of polar crystals from small molecules $[18,19]$. It appears that the assembling of molecules in an antiparallel fashion is generally favored, which explains why polar solids prepared by recrystallization from dilute solutions are quite rare.

While the polar nature of $A$-amylose single crystals was deduced from the 3D model that involves the parallel packing of double helices, it is yet to be confirmed experimentally. The polarity of other polysaccharide crystals has already been investigated by TEM imaging and electron diffraction in the case of native cellulose I and $\beta$-chitin microfibrils. All methods were based on the selective labeling of the polysaccharide reducing end, the crystals being made of parallel chains. Koyama et al. have grown electron-dense silver nanoparticles on the reducing end of cellulose I nanocrystals [20]. Another method was described by Kim et al. who labeled the aldehyde groups at the reducing end of cellulose I microfibrils with colloidal gold nanoparticles [21]. More recently, Imai et al. grafted biotin on the reducing end of $\beta$-chitin nanocrystals [22]. Biotin was then labeled by streptavidin-functionalized gold nanoparticles. All these methods involved incubations at temperature of $50-65^{\circ} \mathrm{C}$. They may be adapted to the case of A-amylose crystals but it would be first necessary to test the stability of the crystals dispersed in an acetone-free aqueous medium, in particular during incubations at $50-60{ }^{\circ} \mathrm{C}$. It may thus be necessary to incubate the crystals at a lower temperature but during longer times.

The labeling methods previously described allowed to unambiguously determine the direction of in vivo biosynthesis of cellulose I and $\beta$-chitin and, in both cases, it was shown that the microfibrils were biosynthesized by addition of glucosyl units on the non-reducing end of the individual polysaccharide chains $[20,22]$. The case of starch is different in the sense that the biosynthesized macromolecules are assembled into a microfibrillar form. In particular, according to the accepted cluster model of amylopectin (the branched glucan and main constituent of starch that is partially crystalline in native granules), one macromolecule only has one reducing end and a very large number of non-reducing ends corresponding the end 
of the short linear branches [23]. The in vivo biosynthesis of amylopectin is currently described by the coordinated action of three enzyme activities (elongation, branching, debranching) [24]. During the branch elongation, the addition of glucosyl units occurs on the non-reducing end of the linear chains [25]. Considering this divergent synthesis of amylopectin, one can assume that the short branches, that would later associate to form crystalline lamellae (with A- or Btype, depending on the botanical source), would thus have their non-reducing end oriented toward the surface of the growing granule [1]. However, the radial development of starch granules in the direction of the non-reducing end of the chains has not been demonstrated experimentally, mostly because of the difficulty to probe the complex ultrastructure of starch granules at a local scale.

The combination of the electron crystallography analysis of in vitro single crystals and the molecular model proposed by Popov et al. [7] led us to conclude that A-amylose single crystals axially grew in vitro in the direction of the reducing end of the chains whereas amylopectin would be biosynthesized outward in native starch granules, in the direction of the non-reducing end.

The results obtained from A-amylose can be compared to those reported for other polysaccharide crystals grown in vitro from dilute solutions: cellulose II [10] and IV [11], inulin [26], chitosan [27], $\beta(1,4)-x y l a n$ [28], dextran [29] and mannan I [30]. In all cases, it has been shown that the constituting molecules were organized in an antiparallel fashion. In addition, several works on single helical V-amylose have proposed antiparallel packings of single helices, which resulted from two kinds of observations. On the one hand, chain-folding is involved when the amylose chains are longer than the thickness of the lamellar crystals $[31,32]$. On the other hand, the space groups deduced from the symmetries of the electron diffraction patterns of the single crystals have generally imposed packings of antiparallel chains [33-37].

The case of A-amylose crystals, made of parallel chains, thus appears to be rather unique among recrystallized polysaccharides. Only B-amylose was also described as a hexagonal packing of 6 -fold parallel double helices $[38,39]$. Lamellar single crystals of B-amylose are extremely difficult to prepare [3] and so far, since it has not been possible to grow single crystals as large as those of A-amylose for X-ray microdiffraction study, a detailed molecular model of $B$-amylose is yet to be proposed.

\section{Acknowledgment}

This research project has been partly funded by a Marie Curie Early Stage Research Training Fellowship of the European Community's $6^{\text {th }}$ Framework Programme, under contract $n^{\circ}$ MEST-CT-2004-503322. We thank P.-C. Escalier (LISBP) for his help in preparing the fractions of synthetic amylose, and D. Dupeyre (CERMAV) for assistance with scanning electron microscopy. The FEG-SEM observations have been performed during a test session organized by JEOL at Consortium des Moyens Technologiques Communs de Grenoble. 


\section{References}

[1] Buléon A, Potocki-Véronèse G, Putaux J-L. Aust J Chem 2007;60:706-18.

[2] Wu HCH, Sarko A. Carbohydr Res 1978;61:27-40.

[3] Buléon A, Duprat F, Booy FP, Chanzy H. Carbohydr Polym 1984;4:161-73.

[4] Imberty A, Chanzy H, Pérez S, Buléon A, Tran V. Macromolecules1987;20:2634-36.

[5] Imberty A, Chanzy H, Pérez S, Buléon A, Tran V. J Mol Biol 1988;201:365-84.

[6] Popov D, Burghammer M, Buléon A, Montesanti N, Putaux J-L, Riekel C. Macromolecules 2006;39:3704-6.

[7] Popov D, Buléon A, Burghammer M, Chanzy H, Montesanti N, Putaux J-L, Potocki-Véronèse G, Riekel C. Macromolecules 2009;42:1167-74.

[8] Montesanti N, Véronèse G, Buléon A, Escalier P-C, Kitamura S, Putaux J-L, Biomacromolecules 2010;11:3049-58.

[9] Cerius ${ }^{2}$ program from Accelrys Inc, San Diego, Ca.

[10] Buléon A, Chanzy H. J Polym Sci Part B Polym Phys 1978;16:833-39.

[11] Buléon A, Chanzy H. J Polym Sci Part B Polym Phys 1980;18:1209-17.

[12] Helbert W, Sugiyama J. Cellulose 1998;5:113-22.

[13] Tsuji M, Kohjiya S. Prog Polym Sci 1995;20:259-308.

[14] Holmes DF, Chapman JA, Prockop D, Kadler KE. Proc Natl Acad Sci USA 1992;89:9855-9.

[15] Gobeaux F, Mosser G, Anglo A, Panine P, Davidson P, Giraud-Guille M-M, Belamie E. J Mol Biol 2008;376:1509-22.

[16] Prockop DJ, Fertala A. J Struct Biol 1998;122:111-8.

[17] Orgel JPRO, Irving TC, Miller A, Wess TJ. Proc Natl Acad Sci USA 2006;103:9001-5.

[18] Hulliger J, Langley PJ, König O, Rothz SW, Quintely A, Rechsteinery P. Pure Appl Opt 1998;7:221-27.

[19] Jouaiti A, Hosseini MW, Kyritsakas N. Chem Commun 2002;17:1898-99.

[20] Koyama M, Helbert W, Imai T, Sugiyama J, Henrissat B. Proc Natl Acad Sci USA 1997;94:9091-5.

[21] Kim U-J, Kuga S, Wada M, Okano T, Kondo T. Biomacromolecules 2000;1:488-92.

[22] Imai T, Watanabe T, Yui T, Sugiyama J. FEBS Lett 2002;510:201-5.

[23] Robin J-P, Mercier C, Duprat F, Charbonnière R, Guilbot A. Cereal Chem 1974;51:389_ 406.

[24] Ball S, Guan HP, James M, Myers A, Keeling P, Mouille G, Buléon A, Colonna P, Preiss J. Cell 1996;86:349-52.

[25] Ball SG, Morell MK. Annu Rev Plant Biol 2003;54:207-33.

[26] André I, Mazeau K, Tvaroska I, Putaux J-L, Winter WT, Taravel F, Chanzy H. Macromolecules 1996;29:4626-35. 
[27] Mazeau K, Winter WT, Chanzy H. Macromolecules 1994;27:7606-12.

[28] Chanzy H, Dubé M, Marchessault RH. Polymer 1979;20:1037-9.

[29] Guizard C, Chanzy H, Sarko A. Macromolecules 1984;17:100-7.

[30] Chanzy H, Pérez S, Miller DP, Paradossi G, Winter W. Macromolecules 1987;20:2407-13.

[31] Manley R St J. J Polym Sci A 1964;2:4503-15.

[32] Yamashita Y. J Polym Sci A 1965;3:3251-60.

[33] Buléon A, Delage M-M, Brisson J, Chanzy H. Int J Biol Macromol 1990;12:25-33.

[34] Helbert W, Chanzy H. Int J Biol Macromol 1994;16:207-13.

[35] Hulleman SHD, Helbert W, Chanzy H. Int J Biol Macromol 1996;18:115-22.

[36] Cardoso MB, Putaux J-L, Nishiyama Y, Helbert W, Hÿtch M, Silveira NP, Chanzy H. Biomacromolecules 2007;8:1319-26.

[37] Nishiyama Y, Mazeau K, Morin M, Cardoso MB, Chanzy H, Putaux J-L. Macromolecules 2010;43:8628-36.

[38] Imberty A, Perez S. Biopolymers 1988;27:1205-21.

[39] Takahashi Y, Kumano T, Nishikawa S. Macromolecules 2004;37:6827-32. 


\section{Tables and Figures}

Table 1. Comparison of the unit cell parameters and volume $V$ of $A$-amylose calculated from the data found in various published works. The experimental data have been collected using different diffraction techniques, at different temperatures $T$.

\begin{tabular}{|c|c|c|c|c|c|}
\hline & $\begin{array}{l}\text { X-rays } \\
\text { (fibers) }^{\mathrm{a}}\end{array}$ & $\begin{array}{c}\text { X-rays } \\
\text { (powder) }^{b}\end{array}$ & $\begin{array}{c}\text { electrons } \\
\text { (single crystals) }^{c}\end{array}$ & $\begin{array}{c}\text { X-rays } \\
\text { (single crystals) }^{d}\end{array}$ & $\begin{array}{l}\text { electrons } \\
\text { (this work) }\end{array}$ \\
\hline$a(\mathrm{~nm})$ & 2.133 & 2.124 & 2.076 & 2.083 & 2.098 \\
\hline$b(\mathrm{~nm})$ & 1.190 & 1.172 & 1.116 & 1.145 & 1.147 \\
\hline$c(\mathrm{~nm})$ & 1.052 & 1.069 & 1.045 & 1.058 & 1.062 \\
\hline$\alpha\left(^{\circ}\right)$ & 90 & 90 & 90 & 90 & 90 \\
\hline$\beta\left(^{\circ}\right)$ & 90 & 90 & 90 & 90 & 90 \\
\hline$\gamma\left(\left(^{\circ}\right)\right.$ & 123.91 & 123.5 & 122.51 & 122.02 & 121.46 \\
\hline$V\left(\mathrm{~nm}^{3}\right)^{\mathrm{e}}$ & 2.216 & 2.218 & 2.042 & 2.139 & 2.180 \\
\hline$T\left({ }^{\circ} \mathrm{C}\right)$ & 20 & 20 & -173 & -173 & -180 \\
\hline
\end{tabular}

(a) monoclinic parameters calculated from the orthorhombic unit cell proposed by Wu and Sarko [2]; (b) Imberty et al. [4]; ( ${ }^{(c)}$ monoclinic parameters calculated from the orthorhombic unit cell proposed by Imberty et al. [5]; (d) Popov et al. [7]; ${ }^{(e)}$ volume of the monoclinic unit cell: $V=a \cdot b \cdot c \cdot \sin \gamma$. 

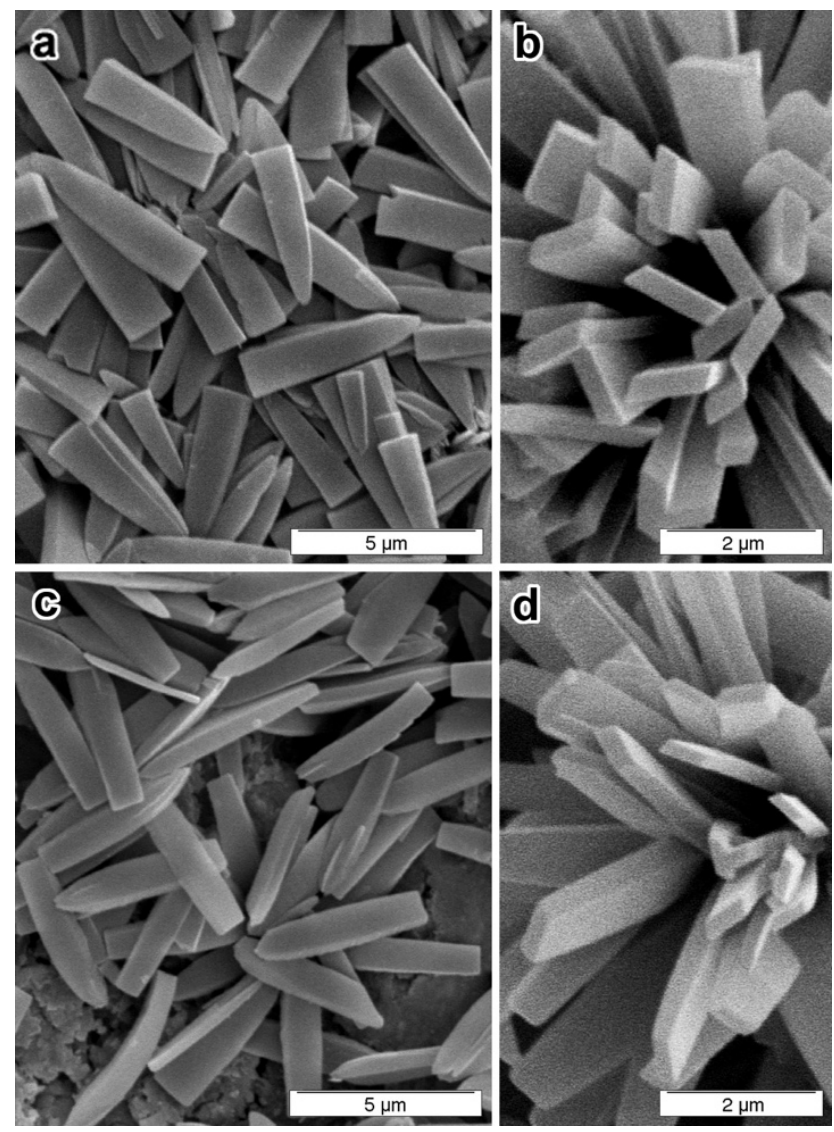

Figure 1. SEM images of A-amylose single crystals prepared from fractions $\operatorname{SAA} 12-23(a, b)$ and SAA17-28 (c,d). In b and d, the crystals are organized in rosettes and their parallelogram cross section can clearly be seen. 

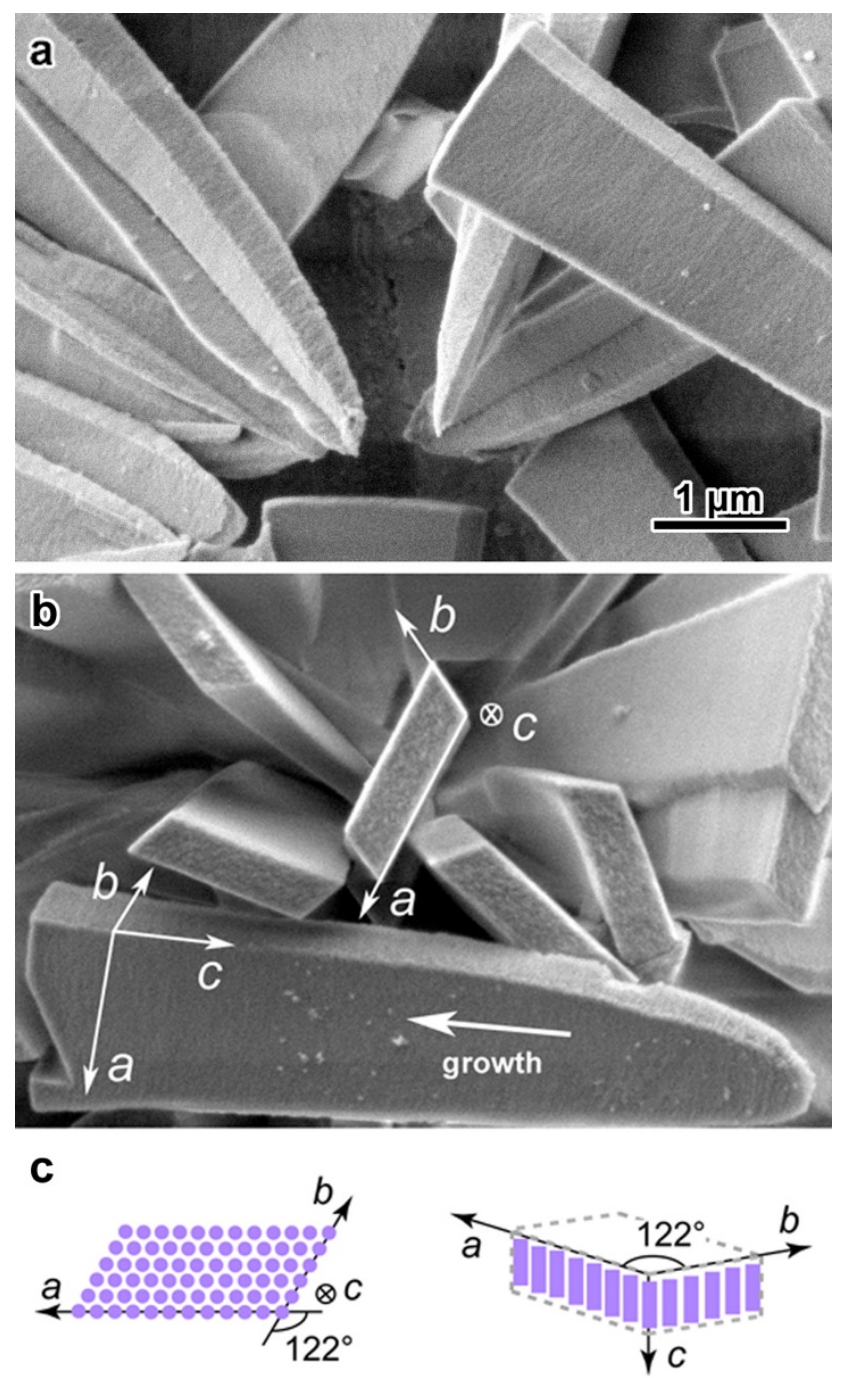

Figure 2. $a, b)$ FEG-SEM images of single crystals prepared from fraction SAA12-23. In b, the main axes of the monoclinic unit cell have been superimposed to crystals in different orientations. c) Schematic representation of the amylose double helices in the monoclinic unit cell. The discs (left) and rods (right) correspond to end-on and edge-on views, respectively, of the double helices. 


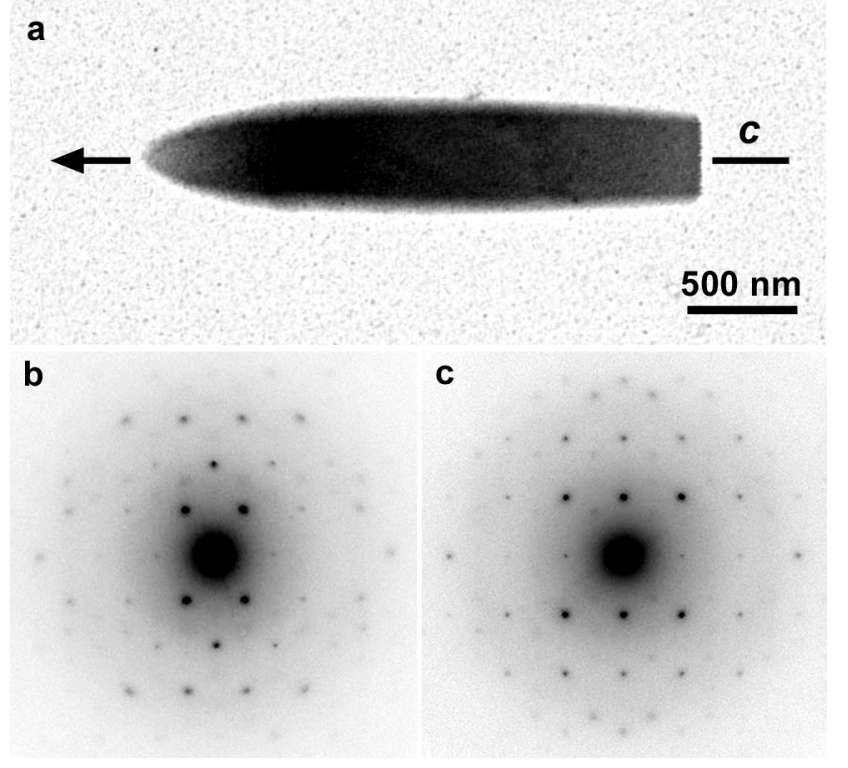

Figure 3. a) TEM image of an A-amylose single crystal prepared from fraction SAA17-28; b,c) most frequently observed electron diffraction patterns collected from untilted single crystals lying flat on the carbon film, correctly oriented with respect to the crystal in image (a). According to the indexation proposed by Imberty et al. [5], the zone axes are [130] (b) and [140] (c), respectively. 


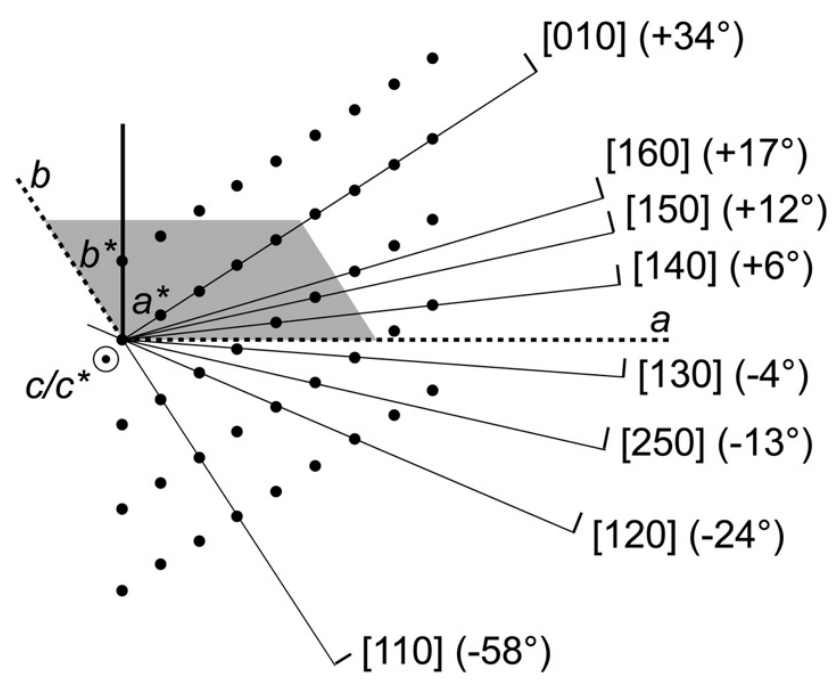

Figure 4. Projection of the reciprocal lattice (black dots) of the monoclinic unit cell of A-amylose along the $c / c^{*}$-axis. The crystal cross-section in real space (in grey) was superimposed to the reciprocal lattice. The $a$ and $b$ axes are indicated by the dotted lines. The dense planes of the reciprocal lattice are indicated by continuous lines. The Miller indices correspond to the axis perpendicular to the selected lattice planes. The tilt angles allowing to bring the zone axis parallel to the incident electron beam are indicated in parentheses. 
a

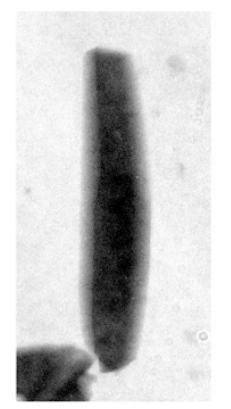

$-20^{\circ}$

b
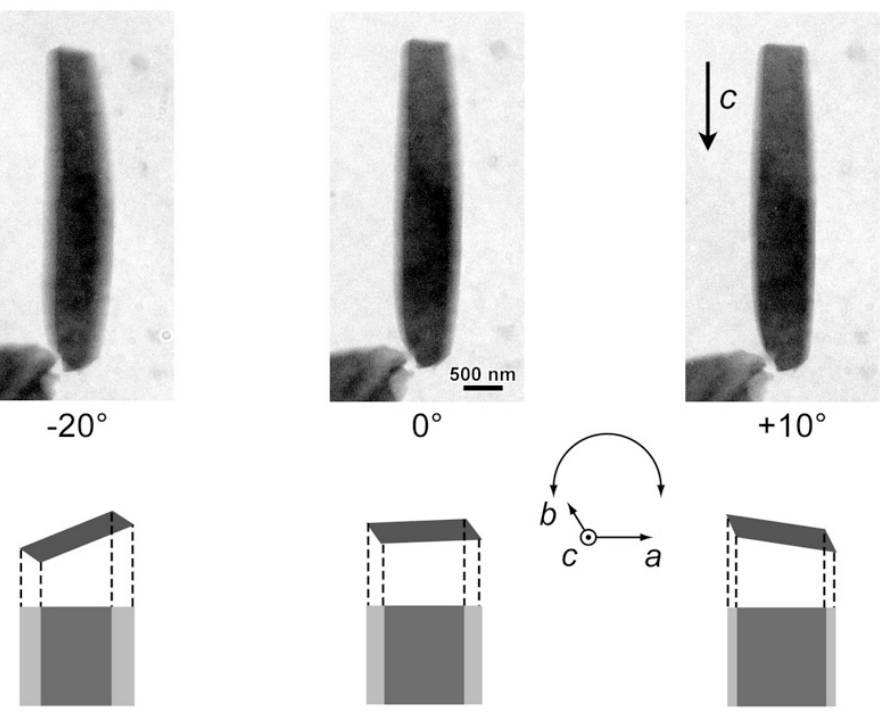

$+10^{\circ}$

$+28^{\circ}$

$0^{\circ}$
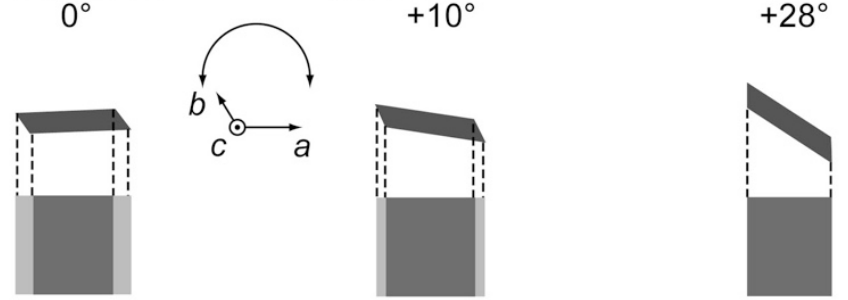

C $\quad[120]\left(-24^{\circ}\right)$
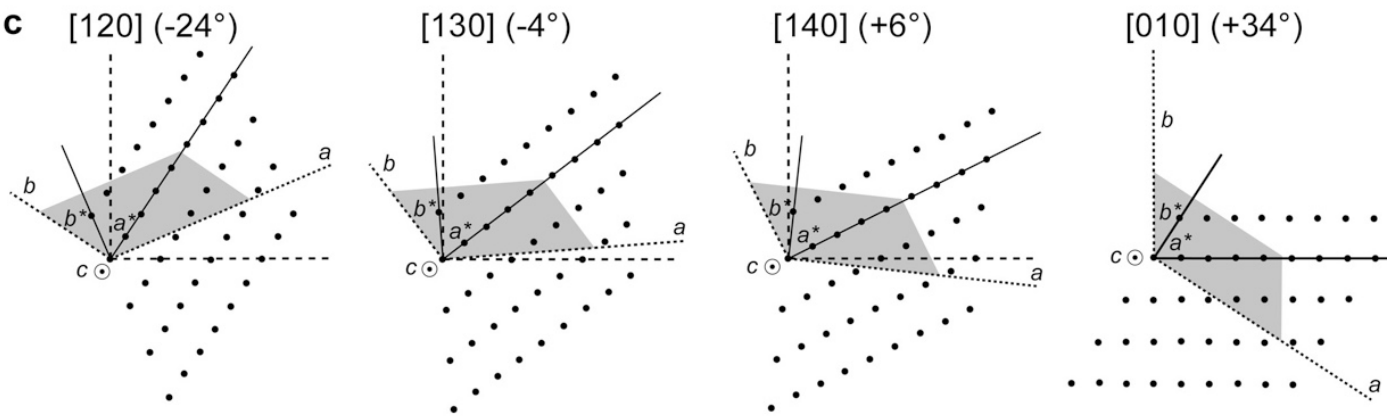

Figure 5. Relationship between the variation of contrast in the TEM images of an A-amylose single crystal tilted around its long axis and the tilt angle: a) TEM images of the crystal in longitudinal view tilted around the $c$-axis. The tilt angles are those of the specimen holder. b) Tilt around the $c$-axis of the crystal seen in cross section and its corresponding longitudinal projection. For simplicity, only two gray levels have been used to distinguish between the edges and the center of the crystal. c) Corresponding projections of the reciprocal lattice (black dots) along the tilt axis $c$. The zone axes and theoretical tilt angles are those defined in Figure 4. The crystal cross section is superimposed in grey. 

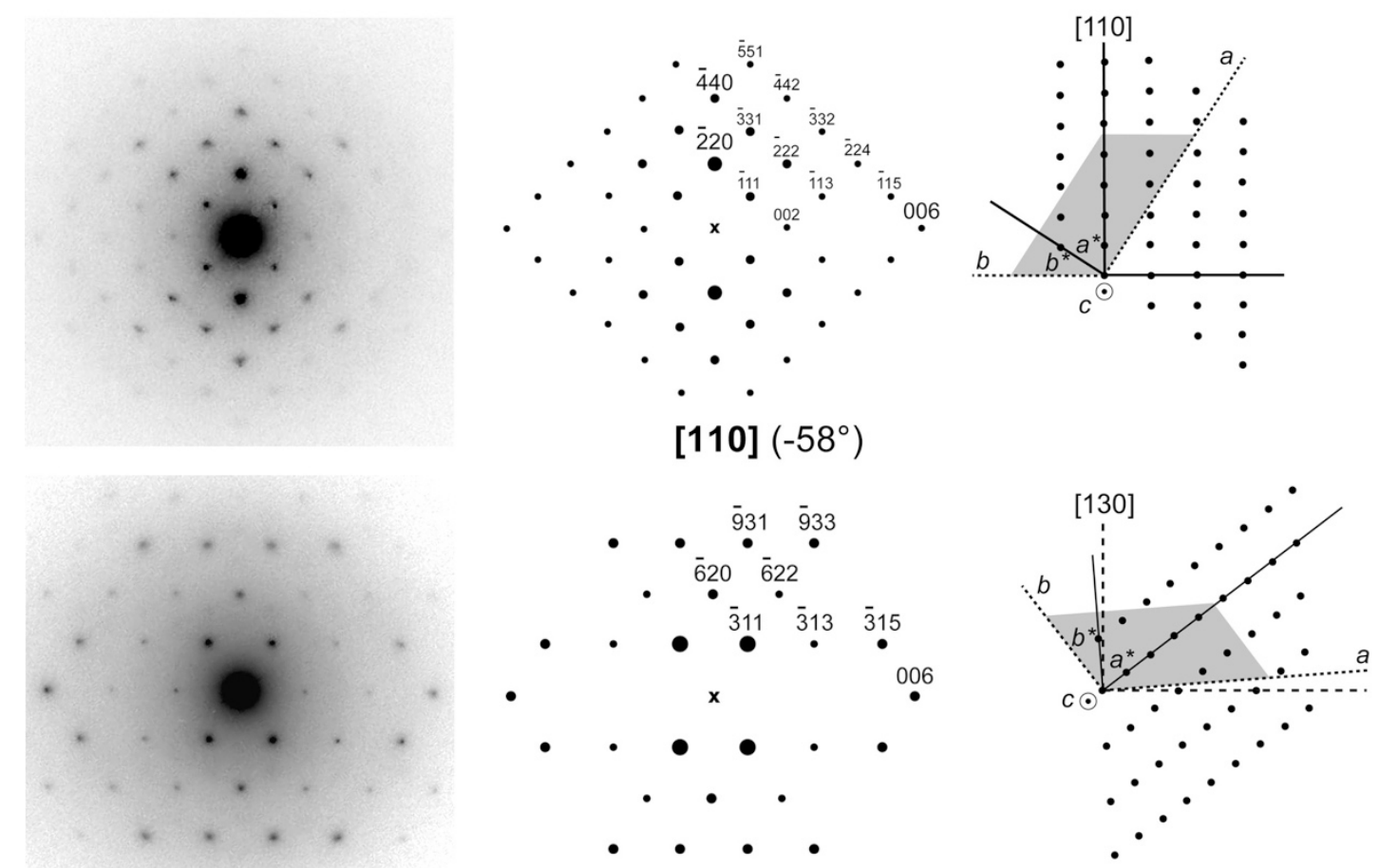

$[130]\left(-4^{\circ}\right)$
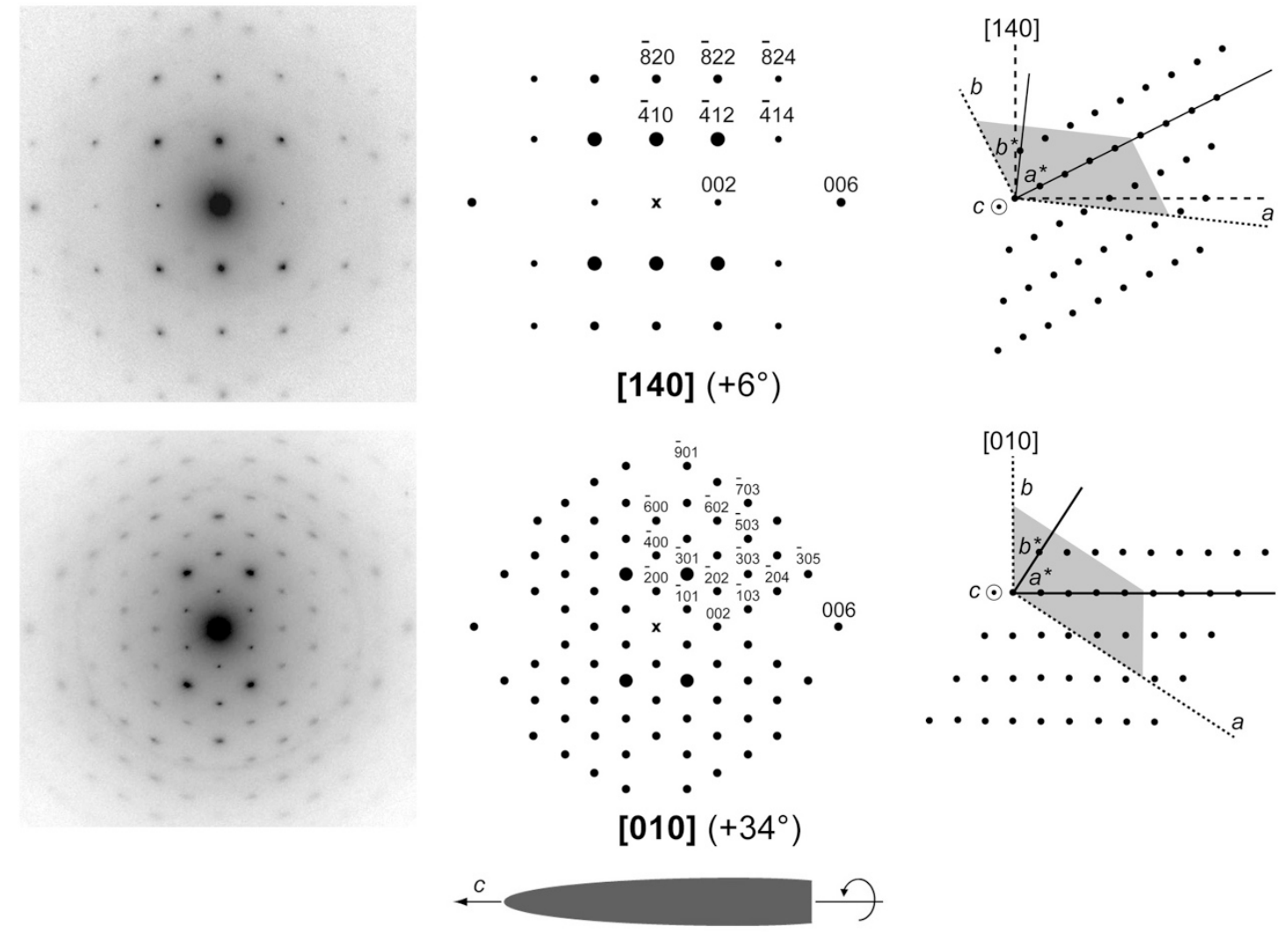

Figure 6. Four typical electron diffraction diagrams (left column) from the [hk0] series of zone axes listed Figure 4, recorded by tilting the crystals about their long axis, horizontally oriented with respect to the diagrams. The indexed patterns are given in the middle column. The projections of the reciprocal lattice (black dots) along the tilt axis $c$ are shown in the right column with the cross section of the crystal superimposed in grey. The tilt angles are defined with respect to the plane that contains the a-axis. 


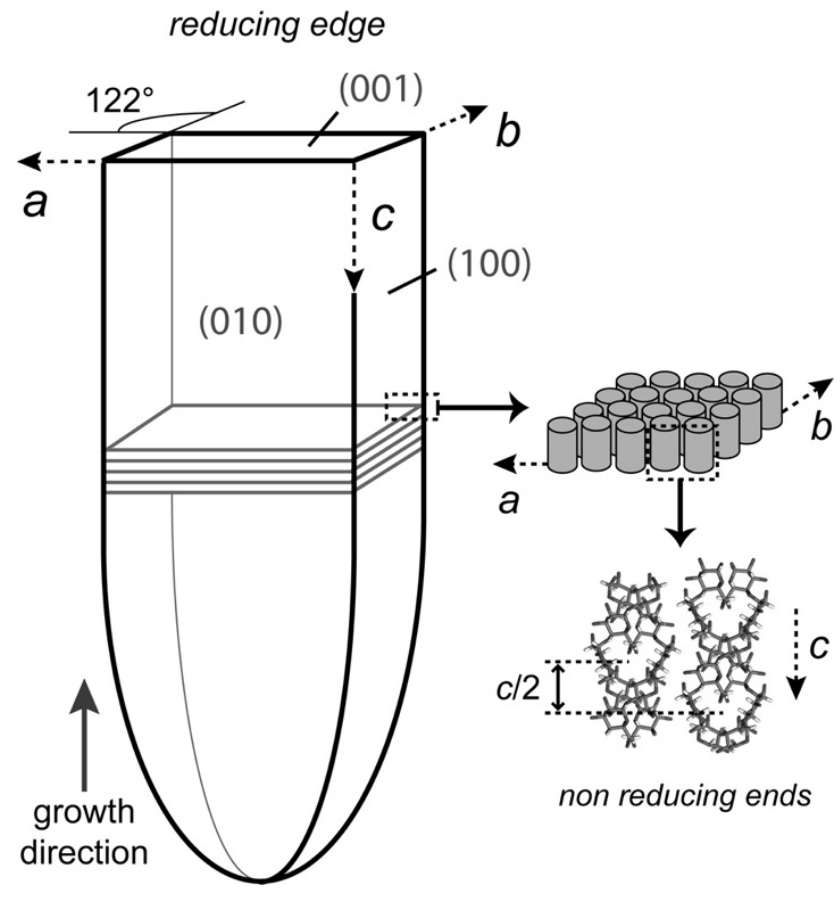

Figure 7. Scheme describing the orientation of the double helices in the long A-amylose single crystals. The grey cylinders correspond to amylose double helices. The corresponding molecular models of two double helices shifted by $c / 2$ with respect to one another have also been drawn, allowing to localize the reducing end of the amylose chains. 


\section{Supplementary Material}

Determination of $\overline{D P_{n}}$ and $\overline{D P_{w}}$ from the high-performance anion-exchange chromatography data (HPAEC):

The characterization of the amylose chains contained in the SAA fractions was performed by HPAEC with pulsed amperometric detection (PAD). The operating conditions were described in details by Montesanti et al. (2010). For each $D P_{l}$, the concentration of maltooligosaccharides $C_{i}$ was evaluated using the linear relationship between the detector response per mole of $\alpha(1,4)$ chains and its degree of polymerization [Koch et al., 1998]. The linear curve coefficients were determined using maltooligosaccharide standards with a $D P$ ranging from 2 to 7 and were used to estimate the amounts of longer chains.

Number- and weight-average $D P \mathrm{~s}\left(\overline{D P_{n}}\right.$ and $\overline{D P_{w}}$, respectively) and polydispersity index $P$ were calculated as:

$$
\overline{D P_{n}}=\frac{\sum_{i} C_{i}}{\sum_{i}\left(\frac{C_{i}}{D P_{i}}\right)} \quad ; \overline{D P_{w}}=\frac{\sum_{i}\left(C_{i} \times D P_{i}\right)}{\sum_{i}\left(C_{i}\right)} ; P=\frac{\overline{D P_{w}}}{\overline{D P_{n}}}
$$

\section{References:}

- Montesanti N., Véronèse G., Buléon A., Escalier P.-C., Kitamura S., Putaux J.-L., Biomacromolecules 2010, 11, 3049-3058.

- Koch, K., Andersson, R., Aman, P. J. Chromatogr. 1998, 800, 199-206. 
a
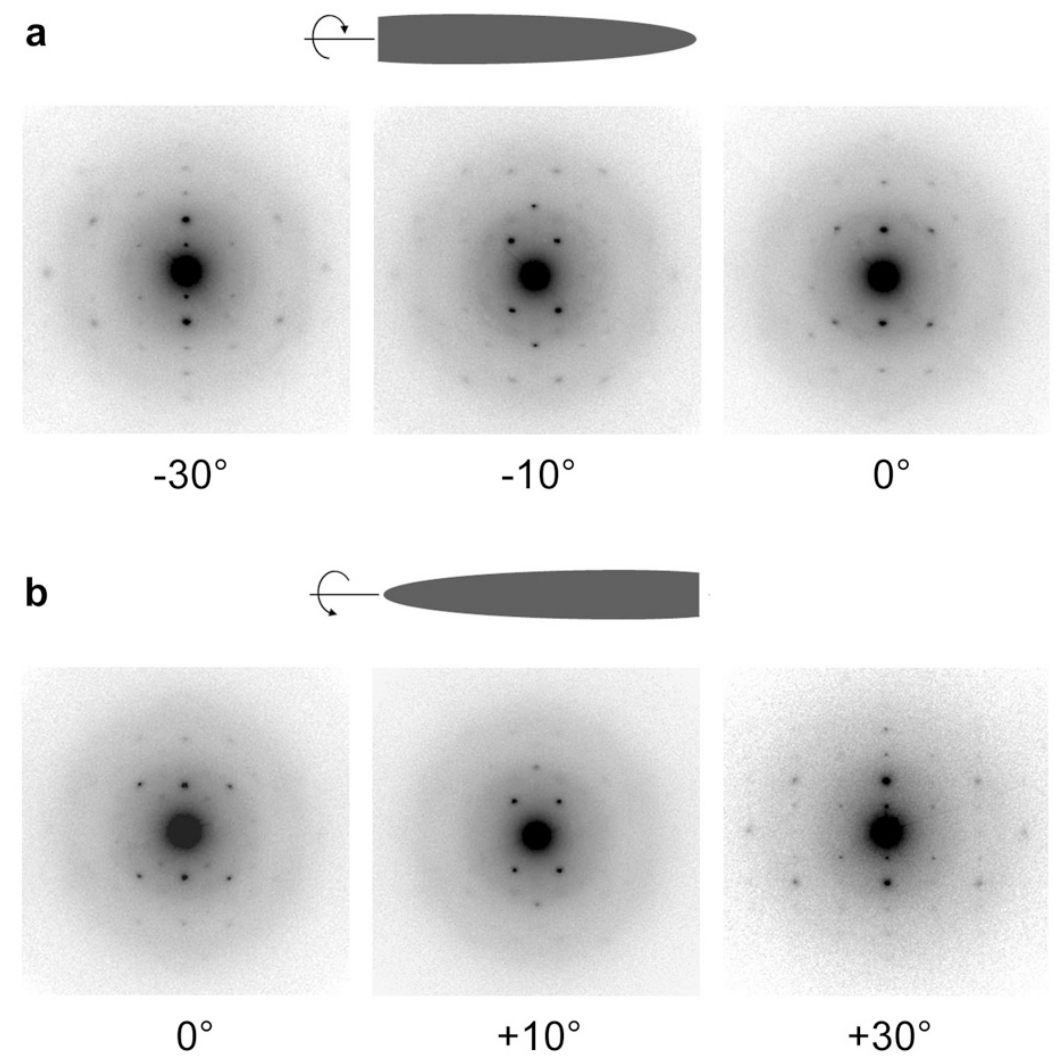

Figure S1. Series of electron diffraction patterns recorded at different crystal tilts around the long axis of two individual crystals oriented in opposite directions. $0^{\circ}$ correspond to the untilted specimen holder. 


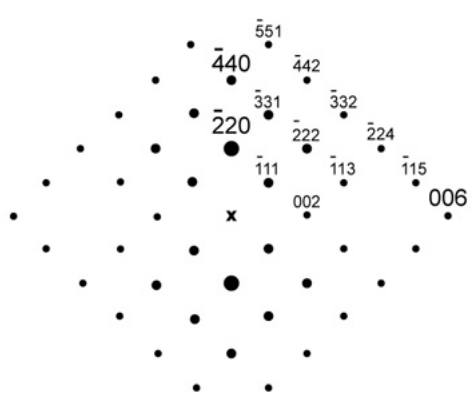

[110] $\left(-58^{\circ}\right)$

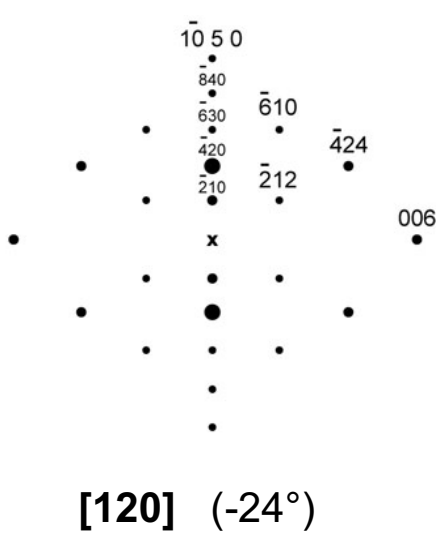

- 10401042

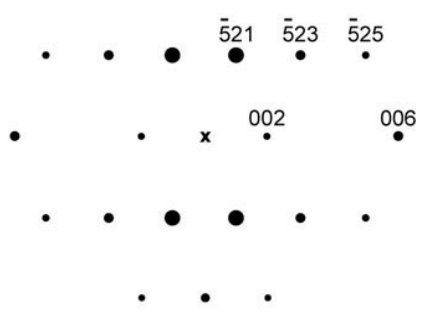

[250] $\left(-13^{\circ}\right)$

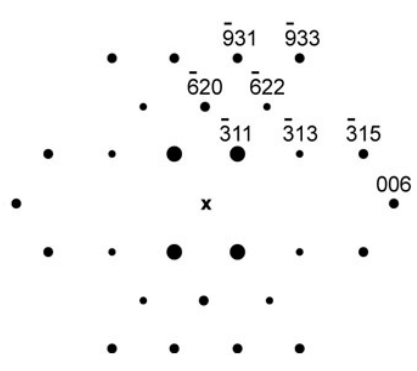

[130] $\left(-4^{\circ}\right)$
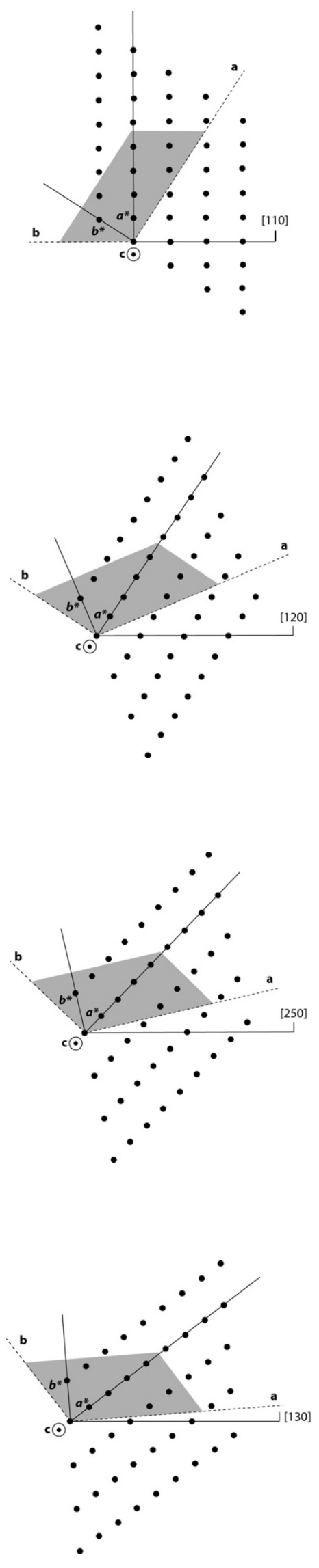

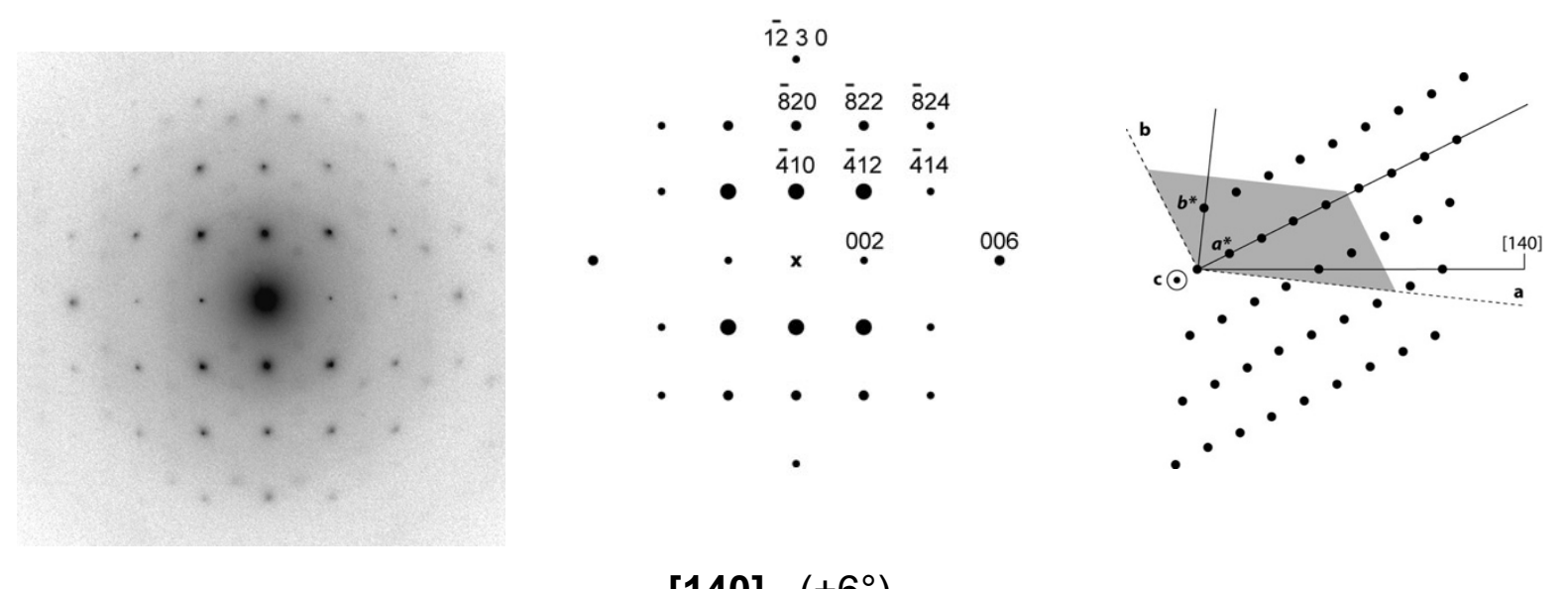

[140] $\left(+6^{\circ}\right)$
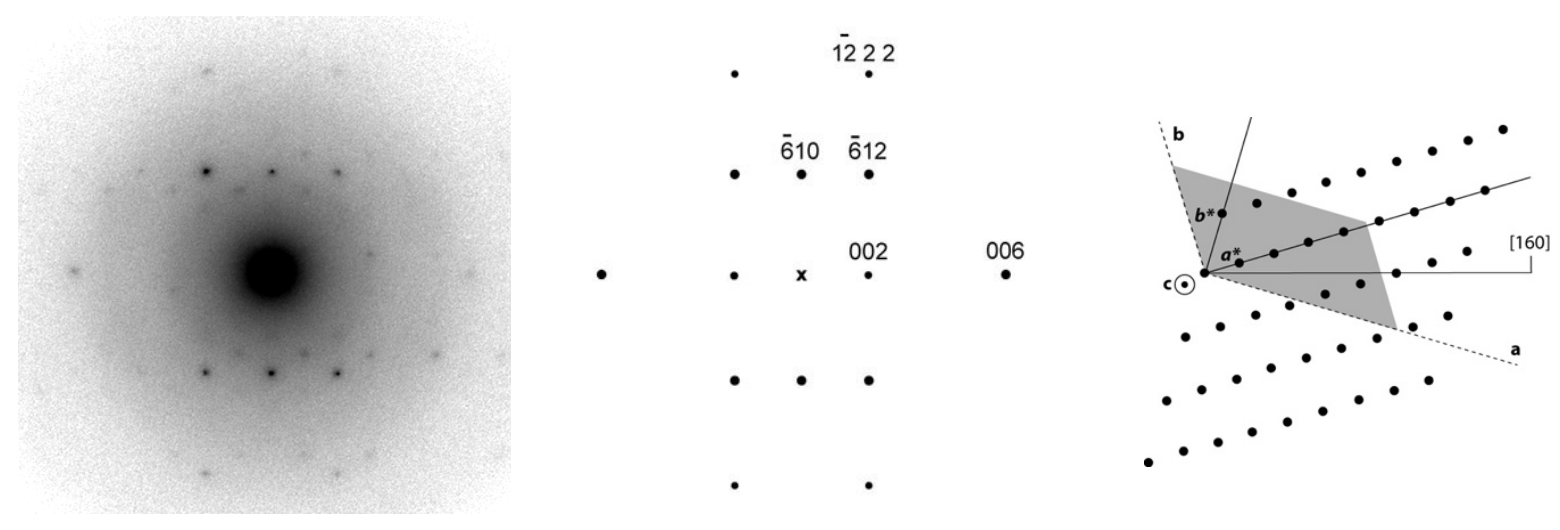

\section{[160] $\left(+16^{\circ}\right)$}
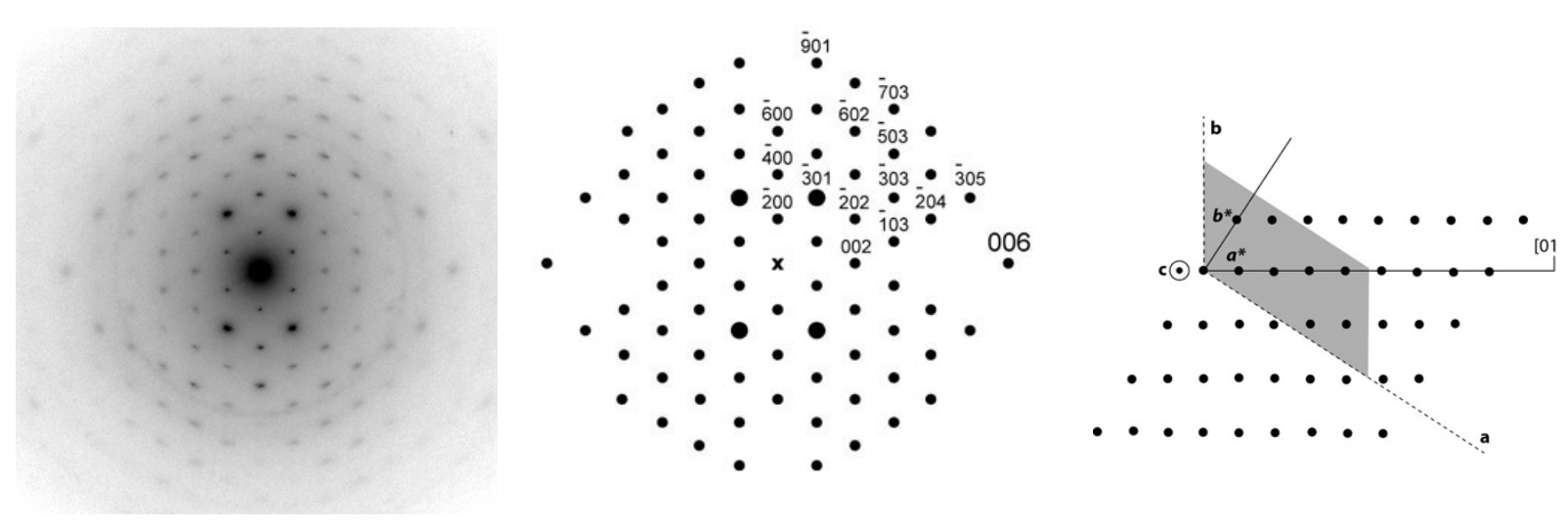

[010] $\left(+34^{\circ}\right)$

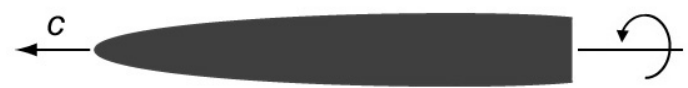

Figure S2. Summary of the electron diffraction diagrams (left column) corresponding to the series of zone axes [hk0] described in the diagram of Figure 4 and recorded by tilting the crystals about their long axis, horizontally oriented with respect to the diffraction patterns. The tilt angle is defined with respect to the horizontal plane that contains the a-axis. The indexed patterns are given in the middle column. In the right column, the crystal cross-section (in gray) has been superimposed on the reciprocal lattice. 


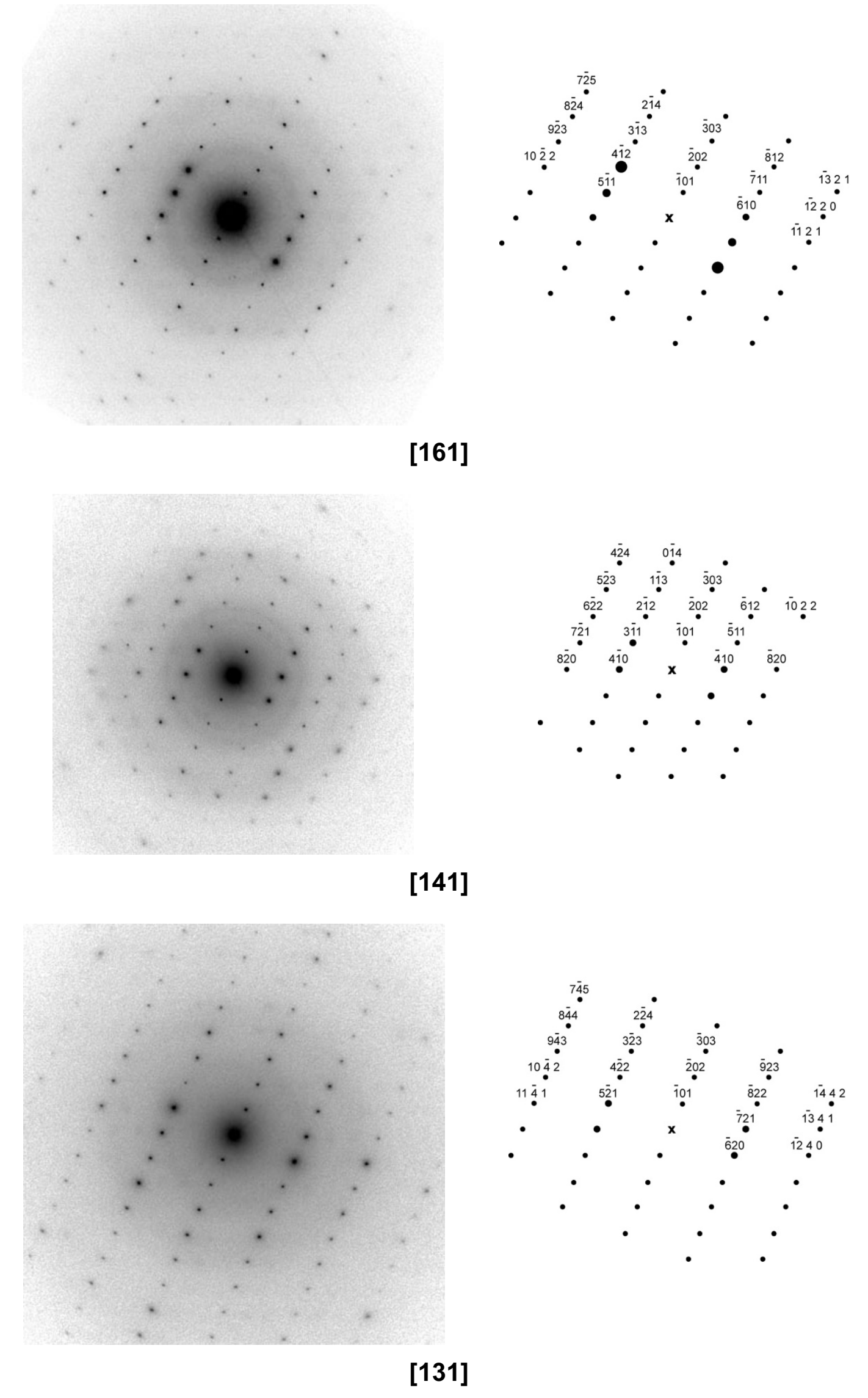



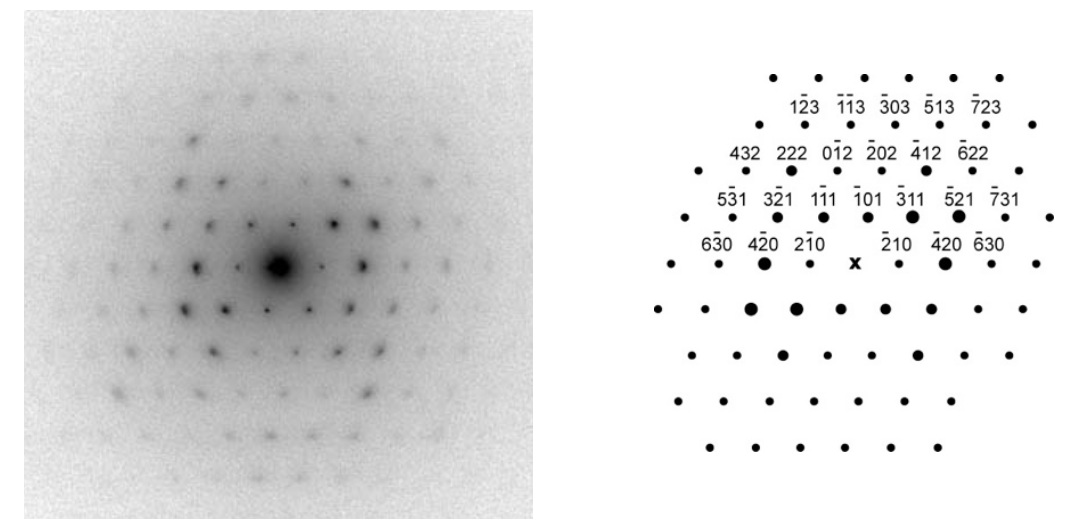

[121]

Figure S3. Electron diffraction patterns (left column) corresponding to the [ $1 k 1]$ series of zone axes recorded from crystals titled around the [ 101$]$ axis. The indexed diagrams are in the right column.
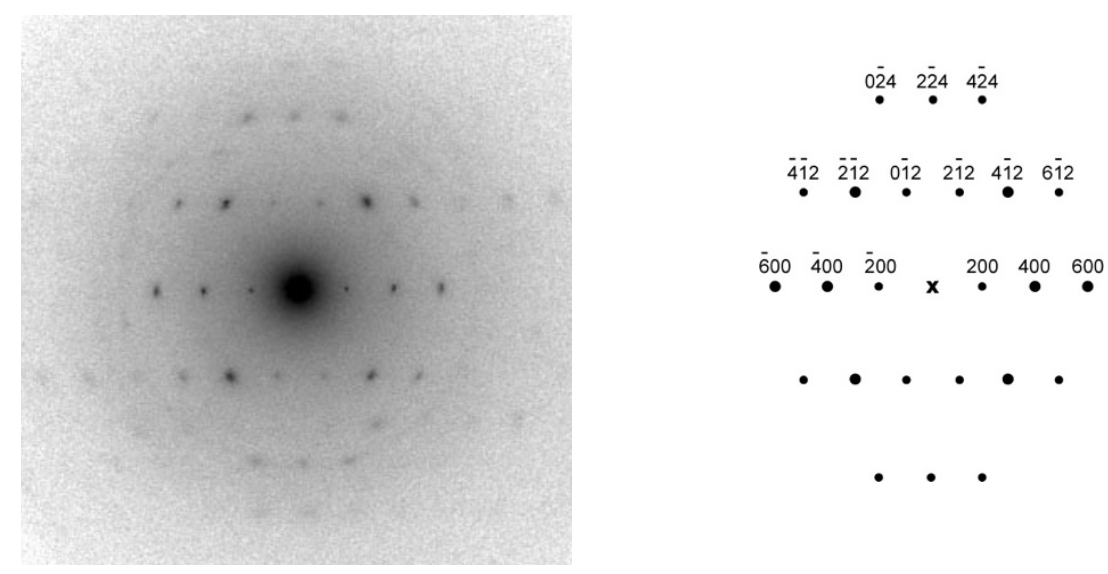

$0 \overline{2} \quad 2 \overline{2} 4 \quad 4 \overline{2} 4$

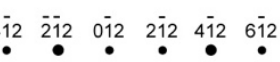

$600 \quad 400 \quad 200 \times 20040060$

[021]
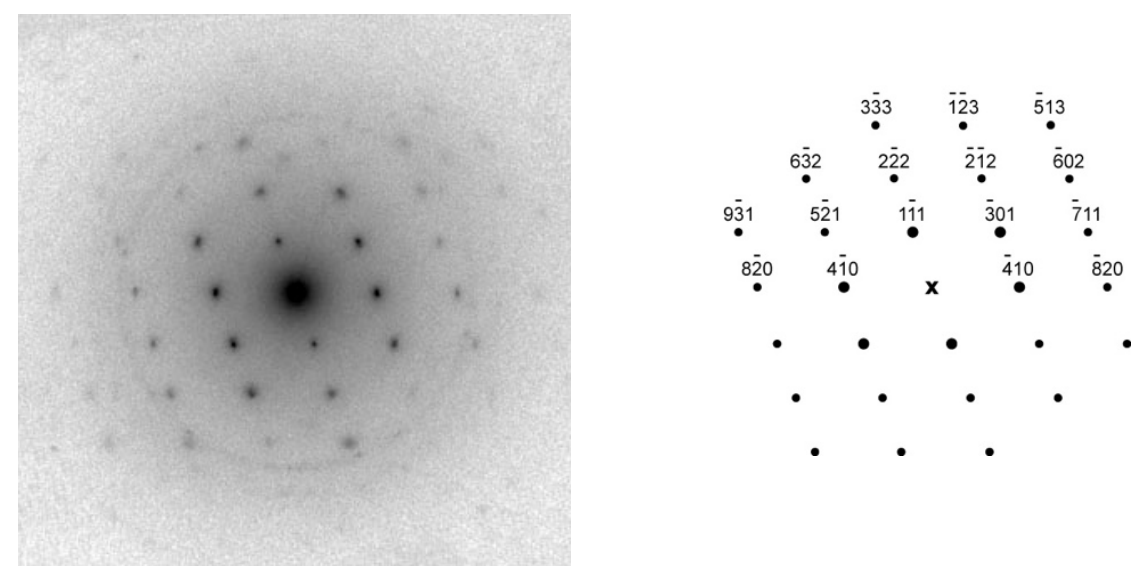

[143]

Figure S4. Two additional diffraction patterns (left column) corresponding to zone axes [021] and [143], respectively. The indexed diagrams are in the right column. The rings correspond to the gold standard. 
Table S1. $h k l$ indices of the set of diffraction spots identified in the diagrams of A-amylose single crystals; experimental $d$-spacings $d_{\text {obs }}$ measured after gold calibration; $d$-spacings $d_{\text {cal }}$ calculated from the unit cell determined with a least-square method; $\left|d_{\text {obs }}-d_{\text {cal }}\right|$ error. The reflections have been sorted by increasing $h$ index.

\begin{tabular}{cccccc}
\hline $\boldsymbol{h}$ & $\boldsymbol{k}$ & $\boldsymbol{I}$ & $\boldsymbol{d}_{\text {obs }}(\mathbf{n m})$ & $\boldsymbol{d}_{\text {cal }}(\mathbf{n m})$ & $\left|\boldsymbol{d}_{\text {obs }}-\boldsymbol{d}_{\text {cal }}\right|(\mathbf{n m})$ \\
\hline-19 & 6 & 1 & 0.11016 & 0.10960 & 0.00056 \\
-18 & 4 & 6 & 0.09657 & 0.09673 & 0.00016 \\
-17 & 4 & 5 & 0.10659 & 0.10625 & 0.00034 \\
-16 & 4 & 4 & 0.11892 & 0.11730 & 0.00162 \\
-15 & 4 & 3 & 0.13021 & 0.12999 & 0.00022 \\
-15 & 2 & 3 & 0.12446 & 0.12449 & 0.00003 \\
-14 & 4 & 2 & 0.14515 & 0.14422 & 0.00093 \\
-14 & 2 & 8 & 0.09782 & 0.09739 & 0.00043 \\
-14 & 2 & 2 & 0.13843 & 0.13837 & 0.00006 \\
-13 & 4 & 1 & 0.16023 & 0.15937 & 0.00086 \\
-13 & 2 & 7 & 0.10882 & 0.10854 & 0.00028 \\
-13 & 2 & 1 & 0.15470 & 0.15369 & 0.00101 \\
-13 & 1 & 7 & 0.10545 & 0.10570 & 0.00025 \\
-12 & 3 & 0 & 0.17195 & 0.17433 & 0.00238 \\
-12 & 2 & 0 & 0.16930 & 0.16943 & 0.00013 \\
-12 & 2 & 6 & 0.12291 & 0.12240 & 0.00051 \\
-12 & 1 & 6 & 0.11863 & 0.11887 & 0.00024 \\
-11 & 2 & 5 & 0.14040 & 0.14002 & 0.00038 \\
-11 & 1 & 5 & 0.13521 & 0.13554 & 0.00033 \\
-11 & 0 & 3 & 0.14798 & 0.14783 & 0.00015 \\
-10 & 2 & 4 & 0.16368 & 0.16294 & 0.00074 \\
-10 & 1 & 4 & 0.15742 & 0.15715 & 0.00027 \\
-10 & 1 & 2 & 0.18011 & 0.18302 & 0.00291 \\
-10 & 0 & 2 & 0.16944 & 0.16959 & 0.00015 \\
-9 & 3 & 3 & 0.19146 & 0.19398 & 0.00252 \\
-9 & 2 & 3 & 0.19467 & 0.19346 & 0.00121 \\
-9 & 1 & 3 & 0.18047 & 0.18586 & 0.00539 \\
-9 & 0 & 9 & 0.10154 & 0.10148 & 0.00006 \\
-9 & 0 & 3 & 0.17301 & 0.17337 & 0.00036 \\
-9 & 0 & 1 & 0.19603 & 0.19545 & 0.00058 \\
-8 & 4 & 0 & 0.23402 & 0.23822 & 0.00420 \\
-8 & 3 & 2 & 0.22742 & 0.23172 & 0.00430 \\
-8 & 2 & 2 & 0.23556 & 0.23460 & 0.00096 \\
-8 & 2 & 0 & 0.25838 & 0.26150 & 0.00312 \\
-8 & 1 & 2 & 0.22534 & 0.22471 & 0.00062 \\
-8 & 0 & 8 & 0.11378 & 0.11417 & 0.00039 \\
-8 & 0 & 4 & 0.17135 & 0.17108 & 0.00027 \\
-8 & 0 & 2 & 0.20541 & 0.20615 & 0.00074 \\
-7 & 4 & 1 & 0.24251 & 0.24838 & 0.00587 \\
-7 & 3 & 1 & 0.27134 & 0.27662 & 0.00528 \\
-7 & 2 & 3 & 0.22381 & 0.22875 & 0.00494 \\
-7 & 2 & 1 & 0.29201 & 0.28845 & 0.00356 \\
-7 & 1 & 1 & 0.27502 & 0.27674 & 0.00172 \\
-7 & 0 & 7 & 0.13016 & 0.13048 & 0.00032 \\
-7 & 0 & 3 & 0.20735 & 0.20726 & 0.00009 \\
-7 & 0 & 1 & 0.24924 & 0.24856 & 0.00068 \\
-6 & 4 & 2 & 0.23612 & 0.24116 & 0.00504 \\
-6 & 3 & 0 & 0.31111 & 0.31763 & 0.00652 \\
-6 & 2 & 2 & 0.28444 & 0.29097 & 0.00653 \\
-6 & 1 & 0 & 0.34271 & 0.33887 & 0.00384
\end{tabular}




\begin{tabular}{|c|c|c|c|c|c|}
\hline-6 & 0 & 6 & 0.15231 & 0.15222 & 0.00009 \\
\hline-6 & 0 & 4 & 0.19859 & 0.19832 & 0.00027 \\
\hline-6 & 0 & 2 & 0.25615 & 0.26005 & 0.00390 \\
\hline-6 & 0 & 0 & 0.29500 & 0.29826 & 0.00326 \\
\hline-5 & 4 & 3 & 0.22031 & 0.22045 & 0.00014 \\
\hline-5 & 3 & 1 & 0.32467 & 0.33054 & 0.00587 \\
\hline-5 & 2 & 1 & 0.37422 & 0.38043 & 0.00621 \\
\hline-5 & 1 & 3 & 0.26355 & 0.26872 & 0.00517 \\
\hline-5 & 0 & 5 & 0.18178 & 0.18267 & 0.00089 \\
\hline-5 & 0 & 3 & 0.25179 & 0.25170 & 0.00009 \\
\hline-5 & 0 & 1 & 0.33724 & 0.33918 & 0.00194 \\
\hline-4 & 3 & 2 & 0.30183 & 0.30442 & 0.00259 \\
\hline-4 & 2 & 0 & 0.47056 & 0.47644 & 0.00588 \\
\hline-4 & 1 & 2 & 0.36987 & 0.37263 & 0.00276 \\
\hline-4 & 1 & 0 & 0.51906 & 0.52301 & 0.00395 \\
\hline-4 & 0 & 4 & 0.22812 & 0.22834 & 0.00022 \\
\hline-4 & 0 & 2 & 0.34034 & 0.34215 & 0.00181 \\
\hline-4 & 0 & 0 & 0.44200 & 0.44740 & 0.00540 \\
\hline-3 & -2 & 9 & 0.11016 & 0.11018 & 0.00002 \\
\hline-3 & 2 & 1 & 0.47681 & 0.48232 & 0.00551 \\
\hline-3 & 1 & 1 & 0.58024 & 0.58194 & 0.00170 \\
\hline-3 & 0 & 5 & 0.19980 & 0.20011 & 0.00031 \\
\hline-3 & 0 & 3 & 0.30463 & 0.30445 & 0.00018 \\
\hline-3 & 0 & 1 & 0.51556 & 0.52011 & 0.00455 \\
\hline-3 & -1 & 5 & 0.18746 & 0.18964 & 0.00218 \\
\hline-2 & -2 & 8 & 0.12446 & 0.12447 & $1.0006 \mathrm{e}$ \\
\hline-2 & 1 & 0 & 0.94523 & 0.95288 & 0.00765 \\
\hline-2 & 0 & 4 & 0.25308 & 0.25455 & 0.00147 \\
\hline-2 & 0 & 2 & 0.45694 & 0.45667 & 0.00027 \\
\hline-2 & 0 & 0 & 0.90550 & 0.89480 & 0.01070 \\
\hline-2 & -1 & 4 & 0.23556 & 0.23789 & 0.00233 \\
\hline-2 & -1 & 2 & 0.37962 & 0.37710 & 0.00252 \\
\hline-1 & 0 & 3 & 0.34632 & 0.34730 & 0.00098 \\
\hline-1 & 0 & 1 & 0.91389 & 0.91335 & 0.00054 \\
\hline-1 & -2 & 3 & 0.27570 & 0.27054 & 0.00516 \\
\hline-1 & -2 & 5 & 0.18800 & 0.18950 & 0.00150 \\
\hline-1 & -1 & 7 & 0.14932 & 0.14843 & 0.00089 \\
\hline-1 & -1 & 3 & 0.31366 & 0.31731 & 0.00365 \\
\hline-1 & -2 & 7 & 0.14242 & 0.14268 & 0.00026 \\
\hline 0 & -1 & 2 & 0.46266 & 0.46672 & 0.00406 \\
\hline 0 & -1 & 6 & 0.17531 & 0.17419 & 0.00112 \\
\hline 0 & -2 & 4 & 0.23034 & 0.23336 & 0.00302 \\
\hline 0 & -2 & 6 & 0.16616 & 0.16645 & 0.00029 \\
\hline 0 & 0 & 2 & 0.52781 & 0.53104 & 0.00323 \\
\hline 0 & 0 & 4 & 0.26461 & 0.26552 & 0.00091 \\
\hline 0 & 0 & 6 & 0.17625 & 0.17701 & 0.00076 \\
\hline 1 & -1 & 1 & 0.77109 & 0.77913 & 0.00804 \\
\hline 1 & -1 & 5 & 0.20955 & 0.20886 & 0.00069 \\
\hline 1 & -2 & 3 & 0.29909 & 0.29778 & 0.00131 \\
\hline 1 & -2 & 5 & 0.19899 & 0.19818 & 0.00081 \\
\hline 1 & -3 & 5 & 0.18127 & 0.18230 & 0.00103 \\
\hline 2 & -1 & 0 & 0.95100 & 0.95288 & 0.00188 \\
\hline 2 & -1 & 2 & 0.46457 & 0.46387 & 0.00070 \\
\hline 2 & -1 & 4 & 0.25605 & 0.25578 & 0.00027 \\
\hline 2 & -2 & 0 & 0.58201 & 0.57323 & 0.00877 \\
\hline 2 & -2 & 2 & 0.38680 & 0.38956 & 0.00276 \\
\hline 2 & -2 & 4 & 0.23956 & 0.24093 & 0.00137 \\
\hline 2 & -4 & 4 & 0.20021 & 0.19111 & 0.00910 \\
\hline 3 & -1 & 1 & 0.57817 & 0.58194 & 0.00377 \\
\hline 3 & -1 & 3 & 0.31462 & 0.31552 & 0.00090 \\
\hline
\end{tabular}




\begin{tabular}{|c|c|c|c|c|}
\hline-3 & 3 & 0.25838 & 0.25971 & 0.00133 \\
\hline-1 & 5 & 0.20317 & 0.20316 & 0.00000 \\
\hline-1 & 7 & 0.14715 & 0.14824 & 0.00109 \\
\hline-2 & 3 & 0.29909 & 0.29630 & 0.00279 \\
\hline-3 & 1 & 0.36022 & 0.35958 & 0.00064 \\
\hline-3 & 3 & 0.25700 & 0.25971 & 0.00271 \\
\hline-4 & 9 & 0.10858 & 0.10899 & 0.00041 \\
\hline-1 & 0 & 0.51784 & 0.52301 & 0.00517 \\
\hline-1 & 2 & 0.37568 & 0.37263 & 0.00305 \\
\hline-1 & 4 & 0.23545 & 0.23676 & 0.00131 \\
\hline-1 & 6 & 0.16672 & 0.16767 & 0.00095 \\
\hline-2 & 0 & 0.47681 & 0.47644 & 0.00037 \\
\hline-2 & 2 & 0.35682 & 0.35463 & 0.00219 \\
\hline-2 & 4 & 0.23400 & 0.23194 & 0.00206 \\
\hline-2 & 8 & 0.12902 & 0.12789 & 0.00113 \\
\hline-4 & 0 & 0.28692 & 0.28660 & 0.00032 \\
\hline-4 & 2 & 0.25114 & 0.25222 & 0.00108 \\
\hline-4 & 8 & 0.12022 & 0.12046 & 0.00024 \\
\hline-2 & 1 & 0.38079 & 0.38043 & 0.00036 \\
\hline-2 & 3 & 0.26792 & 0.26725 & 0.00067 \\
\hline-2 & 5 & 0.18937 & 0.18836 & 0.00101 \\
\hline-2 & 7 & 0.14409 & 0.14219 & 0.00190 \\
\hline-4 & 7 & 0.13338 & 0.13359 & 0.00021 \\
\hline-5 & 1 & 0.22586 & 0.22413 & 0.00173 \\
\hline-1 & 1 & 0.38567 & 0.38472 & 0.00095 \\
\hline-1 & 0 & 0.34114 & 0.33887 & 0.00227 \\
\hline-1 & 2 & 0.28700 & 0.28566 & 0.00134 \\
\hline-1 & 4 & 0.21272 & 0.20900 & 0.00372 \\
\hline-2 & 0 & 0.35002 & 0.34783 & 0.00219 \\
\hline-2 & 2 & 0.28728 & 0.29097 & 0.00369 \\
\hline-2 & 4 & 0.20929 & 0.21106 & 0.00177 \\
\hline-2 & 6 & 0.15971 & 0.15776 & 0.00195 \\
\hline-3 & 0 & 0.31635 & 0.31763 & 0.00128 \\
\hline-3 & 2 & 0.27205 & 0.27259 & 0.00054 \\
\hline-4 & 6 & 0.14775 & 0.14815 & 0.00040 \\
\hline-6 & 0 & 0.19240 & 0.19108 & 0.00132 \\
\hline-2 & 5 & 0.17562 & 0.17330 & 0.00232 \\
\hline-4 & 5 & 0.16368 & 0.16333 & 0.00035 \\
\hline-1 & 2 & 0.22406 & 0.22471 & 0.00065 \\
\hline-2 & 0 & 0.25610 & 0.26150 & 0.00540 \\
\hline-2 & 2 & 0.23126 & 0.23460 & 0.00334 \\
\hline-2 & 4 & 0.18714 & 0.18632 & 0.00082 \\
\hline-4 & 4 & 0.17720 & 0.17732 & 0.00012 \\
\hline-4 & 0 & 0.23783 & 0.23822 & 0.00039 \\
\hline-6 & 9 & 0.09880 & 0.09877 & 0.00003 \\
\hline-4 & 3 & 0.18764 & 0.18724 & 0.00040 \\
\hline-3 & 1 & 0.22260 & 0.22655 & 0.00395 \\
\hline-3 & 3 & 0.19256 & 0.19398 & 0.00142 \\
\hline-3 & 5 & 0.15519 & 0.15663 & 0.00144 \\
\hline-2 & 3 & 0.19661 & 0.19346 & 0.00315 \\
\hline-1 & 2 & 0.18261 & 0.18302 & 0.00041 \\
\hline-2 & 2 & 0.19467 & 0.19236 & 0.00231 \\
\hline-4 & 0 & 0.20757 & 0.20373 & 0.00384 \\
\hline-4 & 2 & 0.19265 & 0.19021 & 0.00244 \\
\hline-4 & 4 & 0.16395 & 0.16163 & 0.00232 \\
\hline-6 & 8 & 0.10511 & 0.10553 & 0.00042 \\
\hline-2 & 1 & 0.18483 & 0.18340 & 0.00143 \\
\hline-4 & 1 & 0.18658 & 0.18521 & 0.00137 \\
\hline-6 & 7 & 0.11241 & 0.11217 & 0.00024 \\
\hline-1 & 2 & 0.15386 & 0.15358 & 0.00028 \\
\hline
\end{tabular}




$\begin{array}{llllll}12 & -2 & 2 & 0.16477 & 0.16142 & 0.00335 \\ 12 & -3 & 0 & 0.17141 & 0.17433 & 0.00292 \\ 12 & -3 & 2 & 0.16298 & 0.16564 & 0.00266 \\ 12 & -4 & 2 & 0.16267 & 0.16528 & 0.00261 \\ 12 & -4 & 4 & 0.14351 & 0.14548 & 0.00197 \\ 12 & -6 & 6 & 0.11835 & 0.11821 & 0.00014 \\ 12 & -4 & 0 & 0.17469 & 0.17391 & 0.00078 \\ 13 & -6 & 5 & 0.12291 & 0.12305 & 0.00014 \\ 14 & -6 & 4 & 0.12638 & 0.12607 & 0.00031 \\ 15 & -6 & 3 & 0.12719 & 0.12681 & 0.00038 \\ 16 & -6 & 2 & 0.12557 & 0.12514 & 0.00043 \\ 17 & -6 & 1 & 0.12230 & 0.12133 & 0.00097 \\ 18 & -6 & 0 & 0.11667 & 0.11594 & 0.00073 \\ & & & & \text { average error } & 0.00192\end{array}$

